\title{
Title \\ Type I IFN promotes pathogenic inflammatory monocyte maturation during H5N1 infection
}

Slim Fourati ${ }^{1}$, David Jimenez-Morales ${ }^{2}$, Judd Hultquist ${ }^{3}$, Max W. Chang ${ }^{4}$, Christopher Benner ${ }^{4}$, Nevan Krogan $^{5,6,7}$, Lars Pache ${ }^{8}$, Sumit Chanda ${ }^{8}$, Rafick-Pierre Sekaly ${ }^{1}$, Adolfo García-Sastre ${ }^{9,10,11,12}$ and Melissa B. Uccellini ${ }^{9,11,13}$

${ }^{1}$ Emory University School of Medicine, Atlanta, GA

2Department of Medicine, Division of Cardiovascular Medicine, Stanford University, Stanford, CA

${ }^{3}$ Division of Infectious Diseases, Northwestern University Feinberg School of Medicine, Chicago, IL

${ }^{4}$ Department of Medicine, University of California San Diego, La Jolla CA

${ }^{5}$ Department of Cellular and Molecular Pharmacology, University of California, San Francisco, CA

${ }^{6}$ Quantitative Biosciences Institute (QBI), University of California, San Francisco, CA

${ }^{7}$ The J. David Gladstone Institutes, San Francisco, CA

${ }^{8}$ Immunity and Pathogenesis Program, Infectious and Inflammatory Disease Center, Sanford Burnham Prebys Medical Discovery Institute, La Jolla, CA

${ }^{9}$ Department of Microbiology, ${ }^{10}$ Department of Medicine, Division of Infectious Diseases, ${ }^{11}$ Global Health and Emerging Pathogens Institute, and ${ }^{12}$ The Tisch Cancer Institute, Icahn School of Medicine at Mount Sinai, New York, NY

${ }^{13}$ Correspondence: P: 212-241-8284, F: 212-534-1684, E: melissa.uccellini@mssm.edu

\section{Abstract}

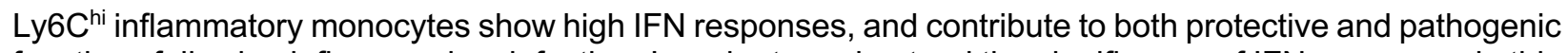
functions following influenza virus infection. In order to understand the significance of IFN responses in this subset, we examined monocytes during infection with a lethal H5N1 virus that induces high levels of IFN and a low-pathogenicity H1N1 virus that induces low levels of IFN. We show that H5N1 infection results in early recruitment of high numbers of Ly6 $\mathrm{C}^{\text {hi }}$ monocytes and induction of chemokines and Ifnb1. Using unbiased transcriptomic and proteomic approaches, we also find that monocytes are significantly enriched during H5N1 infection and are associated with chemokine and IFN signatures in mice, and with severity of symptoms after influenza virus infection in humans. Recruited Ly6C ${ }^{\text {hi }}$ monocytes subsequently become infected in the lung, produce IFN- $\beta$, and mature into $\mathrm{FasL}^{+}$monocyte-derived cells (FasL ${ }^{+} \mathrm{MCs}$ ) expressing dendritic cell markers. Both $\mathrm{Ccr}^{-/-}$and Fas ${ }^{\text {gld }}$ mice are protected from lethal infection, indicating that monocytes contribute to pathogenesis. Global loss of type I and type III IFN signaling in Stat $2^{-/}$mice results in loss of monocyte recruitment, likely reflecting a requirement for IFN-dependent chemokine induction. Here we show that IFN is not directly required for monocyte recruitment on an IFN-sufficient background, but is required for maturation to FasL ${ }^{+} \mathrm{MCs}$. Loss of IFN signaling skews to a Ly6Clo phenotype associated with tissue repair, suggesting that IFN signaling in monocytes is a critical determinant of influenza virus pathogenesis.

\section{Introduction}

Monocytes are hematopoietic cells that develop from myeloid progenitors in the bone marrow and traffic via the bloodstream to peripheral tissues. Chemokine (C-C motif) receptor 2 (CCR2) is required for their release from the bone marrow into the blood, in a process dependent on both chemokine (C-C motif) ligand 2 (CCL2) and CCL7. Recruitment of monocytes from the blood to the tissues is independent of CCR2 and likely involves other chemokines (1-3). Monocytes are defined by expression of CD115 and CD11b, and are divided into two subsets termed "inflammatory" and "patrolling" monocytes. Inflammatory monocytes express high levels of Ly6C and CCR2 in mice, and are CD $14^{+} C C R 2^{+} C D 16$ in humans. Patrolling monocytes express low levels of Ly6C, no CCR2 and are GFPhi in CX3CR1 ${ }^{\text {GFP }}$ mice, and are $\mathrm{CD} 14^{\mathrm{lo}} \mathrm{CX} 3 \mathrm{CR} 1^{\mathrm{hi}} \mathrm{CD} 16^{+}$in humans. Inflammatory signals lead to recruitment of Ly6C ${ }^{\text {hi }}$ monocytes to sites of infection, where they differentiate into various tissue macrophage and dendritic cell populations that aid in infection clearance. Ly6 $\mathrm{C}^{\text {hi }}$ monocytes can give rise to Ly6 $\mathrm{C}^{\mathrm{lo}}$ monocytes (4-7), although development independent of Ly6C $C^{\text {hi }}$ monocytes is also supported by some studies $(8,9)$. The Ly6Clo subset has been associated with wound healing and tissue remodeling following injury or infection (10-12). tissue damage when recruited in excess numbers. Monocyte populations that have differentiated into 
effector cells in the lung have been termed various things during influenza virus infection - including monocyte DCs, TNF-a/iNOS-producing DCs, inflammatory DCs, exudate macrophages, and inflammatory monocyte-macrophages (13-15). Currently there is no naming consensus for these populations, which has led to confusion in the literature. Here we follow the naming convention proposed by Guilliams (16) and refer to monocyte-derived cells differentiated in the influenza-infected lung as 'FasL ${ }^{+} \mathrm{MCs}$ ' to denote that they are monocyte-derived cells (MCs) with the functional characteristic of FasL expression, in addition to DC markers including CD11C and MHC II. Importantly, they express CCR2, CD115 and CD64, and not CD24 indicating monocyte rather than dendritic cell origin. The populations described in different studies likely represent similar, but heterogeneous differentiated MC populations. Monocyte accumulation in the lung has also been described in many infections including SARS-CoV-1 and SARS-CoV-2 (17-19). Influenza-infected $\mathrm{Ccr}^{-/}$mice fail to recruit monocytes, and show delayed viral clearance, decreased T cell accumulation in the draining lymph node, and decreased $\mathrm{CD}^{+} \mathrm{T}$ cell priming $(20,21)$ supporting protective roles for monocytes. However, CCR2 deficiency protects from influenza-induced mortality in most studies $(20,22,23)$, correlating with decreased lung injury - indicating that in many cases monocytes acquire pathogenic phenotypes and contribute to immune-mediated tissue damage.

Type I and type III IFN are critical for restricting viral replication and systemic dissemination, as evidenced by the high susceptibility of Ifnar1 ${ }^{-/}$mice to a number of viruses (24). However, Ifnar1 ${ }^{-/}$mice infected with influenza virus show increased or decreased susceptibility to infection depending on the experimental conditions (25), making the precise role of IFN during influenza virus infection in vivo more difficult to define. This likely reflects the ability of IFN to act in many different ways on a wide-range of cell types, and to induce the expression of ISGs, which orchestrate later immune cell infiltration. Factors that influence the balance between the protective and pathogenic functions of IFN include the background of the mouse strain, the presence or absence of functional $\mathrm{Mx} 1$ expression, the viral strain, and redundancy between the functions of type I and type III IFN. Highly susceptible 129 and DBA/1 strains produce high levels of IFN, which subsequently leads to immune-mediated tissue damage (26). Therefore lack of IFN-signaling during influenza virus infection in these strains is protective. Similar differences in pathogenesis in Ifnar $1^{-/}$mice have been reported for SARS-CoV-1, with IFN mediating detrimental effects in highly susceptible BALB/C mice (17), but not C57BL/6 or 129 mice $(27,28)$. Most inbred mouse strains lack expression of Mx1 (29), however on an Mx1-sufficient background IFN is protective through induction of Mx1 expression and potent restriction of influenza virus replication. Importantly, the increased susceptibility phenotype is only strongly evident in strains lacking both type I and type III IFN signaling due to redundancy in Mx1 induction (30). Certain viral strains have the ability replicate outside of the lung due to ability of the hemagglutinin protein to be cleaved independently of trypsin-like proteases. In these strains, lack of type I IFN signaling is detrimental and leads dissemination of virus outside of the lung (31-34). Given the many different phenotypes of influenza-infected Ifnar $1^{1 /}$ mice, understanding the function of IFN on specific cell types during influenza virus infection will be important for understanding pathogenesis.

Using an ISRE-dependent reporter mouse, we previously showed that Ly6C ${ }^{\text {hi }}$ monocytes have high IFN responses following influenza virus infection (35), however the significance and specific function of IFN in this subset is not known. During influenza virus infection, monocytes have been shown to accumulate in a manner dependent on virus pathogenicity and type I IFN levels $(22,36,37)$. In addition, in susceptible mouse strains, high IFN levels lead to excessive monocyte recruitment. IFN-dependent expression of TRAIL by monocytes subsequently leads to damage to the lung epithelium $(26,38)$. Here we investigated the functional significance of IFN signaling in Ly6 $\mathrm{C}^{\text {hi }}$ monocytes. We show that IFN-signaling is not directly required for recruitment, but is critical for maturation to a pathogenic phenotype. In the absence of IFN signaling monocyte phenotypes are skewed to a Ly6 $\mathrm{C}^{\mathrm{lo}}$ phenotype associated with tissue repair, indicating that IFN signaling in monocytes is critical for determining the balance between the protective and pathogenic functions of type I IFN during influenza virus infection.

\section{Results}

Ly6 $C^{\text {hi }}$ monocytes are associated with H5N1 infection in mice, and severity of symptoms in humans Using an ISRE-dependent reporter mouse for type I and type III IFN ( $\left.M \times 1^{\text {gfp }}\right)$, we previously showed that $\mathrm{Ly}_{6 \mathrm{C}}{ }^{\mathrm{hi}}$ monocytes display very high IFN responses in the lung following influenza virus infection (35). In order to understand the significance of IFN responses in this subset, we examined monocytes during infection with either an avian H5N1 strain of influenza virus (A/Vietnam/1203/04 HALo) or a lab-adapted 
H1N1 infection (A/PR/8/34). The HALo strain has been engineered to remove the hemagglutinin multibasic cleavage site, which limits systemic spread of the virus and allows use under BSL2 conditions, however the virus retains its other virulence determinants (39). At the doses examined, H5N1 induces an early IFN response that is sustained until the animals succumb to infection - while $\mathrm{H} 1 \mathrm{~N} 1$ induces a delayed IFN response, and the animals go on to survive infection (Fig 1A) (35). At day 2 post-infection, mice infected with H5N1 showed a large influx of Ly6Chi monocytes (Fig 1B). In contrast, mice infected with H1N1 showed Ly6 $\mathrm{C}^{\text {hi }}$ monocyte numbers similar to uninfected mice. We were unable to detect consistent viral titers at day 2 post-infection in $\mathrm{H} 5 \mathrm{~N} 1$ or $\mathrm{H} 1 \mathrm{~N} 1$ infected animals, however day 3 post-infection both viruses replicated to high titers in the lung, (Fig 1C) suggesting viral replication did not account for the large differences in monocyte infiltration and infection. Using complementary unbiased transcriptomic and proteomic approaches, we compared the lung tissue of mice infected with the same H5N1 strain to another mild H1N1 strain (A/California/04/09) at 12 hours, or 1, 2, 3 or 4 days post-infection. In order to identify immune cell subsets that may be differentially engaged by $\mathrm{H} 5 \mathrm{~N} 1$ and $\mathrm{H} 1 \mathrm{~N} 1$, we assessed the enrichment (i.e. overlap) between known transcriptomic (40) and proteomic (41) markers of immune cell subsets ( $T$, B, NK, monocyte, $\mathrm{mDC}, \mathrm{pDC}$ ) among genes/proteins differentially expressed between the two strains. Monocytes were the only significantly enriched immune cell subset associated with H5N1 infection by transcriptomics (Fig 1D) and proteomics (Fig S1). Monocytic genes and proteins induced by H5N1 included the cell surface markers Cd14 and Fcgr1a as well as the interleukin II15 and TLR4-ligand S100a9. In order to determine if the monocytes gene signature induced after H5N1 infection in lung of mice can also be detected in blood, we analyze an independent set of mice infected with H3N2 for which both lung and blood RNA-sequencing was performed [PMID:26413862]. The monocytes signatures was induced post-infection and reach it maximum induction 3 days following infection in both lung and blood.

Subsequently, we investigated the use of the monocytes gene signature induced after H5N1 as being associated with influenza disease severity in human in a large meta-analysis of blood transcriptomic datasets [PMID: 30356117]. Fig 2). Similarly to the mice lung, the monocytes gene signature was induced day 2 to day 4 after infection in participants that developed severe symptoms. Genes in the monocyte signature that were most significantly associated with severity in human include the chemokine coding genes CXCL10 and CCL2. These subjects showed heightened expression of the TNF/NFkb confirming the pro-inflammatory environment triggered by the infection. Markers of monocytes (CD86, CCR5) and inflammasome activation (CASP1, NLRP3) required to activate pro-IL-1 $\beta$ and IL-18 were also associated with disease severity. Counterintuitively, this gene signature also included increased expression of the immunomodulatory cytokine IL-10 well known to be expressed by monocytes most probably as a negative feedback loop to overcome the heightened inflammatory environment triggered by infection. Similarly to H5N1 infected mice lung severe symptoms. The signature resolved by day 7 in all infected subjects. Altogether, these results highlight the pro-inflammatory environment triggered by H1N1 infection in human subjects which is largely attributed to the activation of cells of the monocyte lineage. The conservation of these gene signatures between mice and human infected with the flu virus and the association with disease severity suggests that monocyte activation is a hallmark feature of flu infection pathogenesis.

\section{H5N1 infection results in early chemokine and Ifnb1 production}

Ly6C $\mathrm{C}^{\text {hi }}$ monocytes produce the CCR2 ligands CCL2 and CCL7 in an IFN-dependent manner, which further amplifies their recruitment $(17,22)$. Consistent with this, animals infected with H5N1 showed robust induction of $\mathrm{Ccl} / 2$ and $\mathrm{Cc} / 7$, while mice infected with $\mathrm{H} 1 \mathrm{~N} 1$ did not show induction of these chemokines (Fig 1E). Pathway enrichment analysis of the genes and proteins differentially expressed in H5N1 infected mice compared to $\mathrm{H} 1 \mathrm{~N} 1$ infected mice confirmed that $\mathrm{Ccl} 2$ and $\mathrm{Ccl} 7$, as well as a number of other chemokines and cytokines are induced at early timepoints post-infection (Fig 1F).

Transcriptomic analysis also revealed a strong IFN signature at early time points in H5N1 infected mice. In addition, mice infected with H5N1, but not H1N1 showed robust induction of Ifnb1 (Fig 1G), consistent with a report showing Ifnb1 message in sorted $\mathrm{Gr}^{+} \mathrm{CD} 11 \mathrm{~b}^{+}$cells (22). compared to $\mathrm{H} 1 \mathrm{~N} 1$ infected mice (Fig 2C). Interferon-stimulated genes induced in H5N1 infected mice include the viral sensors Dhx58 and Ifih1 and the transcription factor Irf9 (Fig $1 \mathrm{H})$. This is consistent with the reported ability of H5N1 strains to induce a "cytokine storm $(42,43)$. 


\section{Ly6 $\mathrm{C}^{\text {hi }}$ monocytes become infected and produce IFN- $\beta$ during H5N1 infection}

We next examined the phenotype of Ly6 $\mathrm{C}^{\text {hi }}$ monocytes following recruitment to the lung. At day 2 postinfection, H5N1-infected animals showed high numbers of Ly6 $C^{\text {hi }}$ monocytes that were infected as indicated by surface expression of influenza M2 protein, consistent with other reports showing that monocytes can become infected with influenza virus $(22,44)(28555541)$. By day 2 post-infection, H5N1-infected animals showed high frequencies of GFPhi cells in H5N1-infected $M \times 1^{\text {gfp }}$ mice ; H1N1 infected mice showed significantly lower numbers ( 10 fold ) of GFPhi cells than H5N1 infected mice (Fig 3A and 3B). Kinetic analysis showed that H5N1 infected mice showed significancy higher frequencies of We previously observed a lack of Ifnb1 induction in Stat $2^{-/}$mice (35), which was surprising given that Ifnb1 is induced independently of IFN signaling. Lack of Ifnb1 induction in both H5N1-infected Stat2- mice and H1N1 infected WT mice (Fig 2A) correlated with lack of infiltration of Ly6C ${ }^{\text {hi }}$ monocytes, suggesting that these cells might be a primary source of Ifnb1 during infection. In order to determine if Ly6 $\mathrm{C}^{\text {hi }}$ monocytes produce IFN- $\beta$, we infected Ifn $b^{m o b}$ mice, which express YFP from an internal ribosome entry site in the Ifnb1 locus, with H5N1 and monitored cells in the lung for YFP expression. We were able to detect YFP expression in $\sim 18 \%$ of Ly6C ${ }^{\text {hi }}$ monocytes, but not in other cell types including xxxx (Fig 2C), suggesting that Ly6C $\mathrm{C}^{\text {hi }}$ monocytes are indeed the main source of IFN- $\beta$ during influenza virus infection. We were unable to detect YFP expression at various time points post-infection with $\mathrm{H} 1 \mathrm{~N} 1$ (not shown), indicating that IFN- $\beta$ induction during $\mathrm{H} 1 \mathrm{~N} 1$ infection was below the limit of detection of the reporter gene. Ly6C $\mathrm{C}^{\text {hi }}$ monocytes were also GFP in in H5N1-infected Mx $1^{\text {gfp }}$ mice, indicating they both produced, and responded to type I IFN (Fig 3A and $D$ ). Overall the data suggest that during H5N1 infection, Ly6 $\mathrm{C}^{\text {hi }}$ monocytes recruited to the lung become infected and both produce and respond to type I IFN to levels higher than other cell types present in the lung. High levels of type I IFN production by Ly6 $\mathrm{C}^{\text {hi }}$ monocytes in the lung likely contribute to increased infiltration of additional Ly6C ${ }^{\text {hi }}$ monocytes by promoting expression of recruiting chemokines, contributing to a pathological feedback loop.

\section{Ly6C ${ }^{\text {hi }}$ mature into Fas $L^{+} M C s$ during H5N1 infection}

Following recruitment to sites of infection, Ly6C $\mathrm{C}^{\text {hi }}$ monocytes mature into various effector cells with different functional characteristics. When we further examined the phenotype of Ly6C ${ }^{\text {hi }}$ monocytes from H5N1infected mice, we found they expressed higher levels of Ly6C, and upregulated CD11c and MHC Class II (Fig 4A). Influenza infection of 129 mice leads to high levels of IFN production and expression of TRAIL on Ly6C ${ }^{\text {hi }}$ monocytes, leading to subsequent DR5-dependent tissue damage $(23,26)$. We did not detect TRAIL expression on Ly6C hi monocytes during H1N1 or H5N1 infection (not shown). FasL expression has also been reported to be upregulated during influenza virus infection $(45,46)$. When we examined FasL expression on $L y 6 C^{\text {hi }}$ monocytes, we found FasL was upregulated on Ly6C ${ }^{\text {hi }}$ monocytes at day 2 postinfection with H5N1 but not H1N1 (Fig 4A). We observed constitutive Fas expression on both hematopoietic and non-hematopoietic cells (not shown) as reported (45). The data suggest H5N1 infection leads to Ly6C ${ }^{\text {hi }}$ monocyte infection, IFN- $\beta$ production, and maturation to a FasL ${ }^{+} \mathrm{MC}$ phenotype.

In order to determine if our transcriptomic data similarly supported the maturation of infiltrating monocytes, we used CIBERSORT a method that uses gene expression profiles to quantify the distribution of hematopoietic cell susbets based on gene expression profiles. This method infers 22 cell subsets including monocytes, M0 (differentiated from monocytes), M1 (differentiated in LPS/IFN- $\gamma$ ), and M2 (differentiated in LPS/IFN- $\gamma / \mathrm{LL}-4$ ) cells for the monocyte/macrophage lineage. Deconvoluting our transcriptional data using this method, we found an enrichment of "classically activated" M1 macrophages in H5N1-infected mice, which peaked at day 2 post-infection (Fig 4B). Importantly the "M1" classification denotes a proinflammatory phenotype, consistent with our flow cytometry findings, as opposed to the repair phenotype that defines the M2 subset. In order to determine if these M1 macrophages were derived from tissue-resident macrophages, or included mostly infiltrating monocyte-derived macrophages, we performed GSEA enrichment analysis to compare our transcriptomic signatures to known markers of tissue-resident vs. infiltrating macrophages. This analysis revealed the significant enrichment of monocyte-derived macrophage markers in the lungs of H5N1, compared to H1N1 infected mice supporting their origin from infiltrating cells (Fig 4C). Of note these recruited monocytes exhibited heightened expression of several markers of monocyte activation (Cd86, Cd40), markers of the complement pathways (C1), and chemokines including $\mathrm{Ccl} 8$ and $\mathrm{Cxcl} 13$ and as well II10 and Arg2 two cytokines with anti-inflammatory activity reiterating that a negative feedback loop is put in place to counteract potential deleterious effect of the heightened pro-inflammatory response. Of note these cells express also several genes with anti-apoptotic activity (Bcl- 
2 , Birc3) suggesting that they are resistant to cell death signals prevalent in his pro-inflammatory environment

In order to determine if FasL expression was associated with M1 macrophages, we assessed the correlation between the frequency of M1 macrophages post-influenza infection, and cell death-specific pathways. This analysis showed enrichment for genes in the FasL pathway (Fig 4D) and a positive correlation of the FasL pathway with the inferred frequency of M1 macrophages (Fig 4E and Fig S3). FasL pathway was induced after H5N1 infection and reached its peak induction at day 3 post-infection. FasL pathway follow the same kinetics of response to infection as the monocyte signature but delayed by 1 day; this suggest that FasL pathway induction follow the induction of the FasL pathway. To test that FasL pathway is induced more strongly in severe flu disease and follow the induction of the monocytes gene signature, we analysed a publically available transcriptomic dataset of mice infected by two strains of H3N2 (one strain with low virulence and one highly virulent strain) [PMID:21874528]. This analysis confirmed that FasL pathway induction occurs 1 day following induction of the monocytes gene signature (Fig S3A) and revealed that FasL pathway is more strongly induced ( $>3$ fold-difference) by a virulent influenza strain (Fig S3B). The data suggest H5N1 infection is associated with maturation of Ly6C $\mathrm{C}^{\text {hi }}$ monocytes into a proinflammatory $\mathrm{M} 1 / \mathrm{FasL}^{+} \mathrm{MC}$ phenotype

\section{Ly6 $C^{\text {hi }}$ monocytes contribute to lethality during H5N1 infection}

During influenza virus infection, low-level monocyte recruitment is likely beneficial, while excessive recruitment can lead to lung injury. In order to determine whether monocyte recruitment was beneficial, or contributed to lethality during H5N1 infection, we infected WT and $\mathrm{Ccr} 2^{-/-}$mice, which are unable to recruit monocytes from the bone marrow to the blood, with $\mathrm{H} 5 \mathrm{~N} 1$ and monitored survival. $\mathrm{Ccr}^{-1-}$ mice were completely protected following $\mathrm{H} 5 \mathrm{~N} 1$ infection (Fig $5 \mathrm{~A}$ ), suggesting they contribute to lung damage. In addition, Fas/lid mice were partially protected following H5N1 infection (Fig 5B), suggesting that FasL is also a contributing factor to lung damage during H5N1 infection.

\section{IFN-signaling is not directly required for monocyte recruitment}

Using Stat $2^{-/}$mice, we previously reported that Ly6 $\mathrm{C}^{\text {hi }}$ monocyte recruitment is dependent on IFN signaling (35). Similar findings have also been reported in Ifnar $1^{-/}$mice $(22,36)$. Importantly, both Ifnar $1^{-/}$and Stat2${ }^{1}$ - mice fail to induce ISGs including $\mathrm{Cc} / 2$ and $\mathrm{Cc} / 7(35,36)$, which are required for the recruitment of $\mathrm{Ly}_{6 \mathrm{C}}{ }^{\mathrm{hi}}$ monocytes from the bone marrow to the blood. In order to address if IFN-signaling was directly required for Ly6C ${ }^{\text {hi }}$ monocyte recruitment into the blood, we injected WT and Stat $2^{-/}$mice i.v. with CCL7 (47) and measured $\mathrm{Ly} 6 \mathrm{C}^{\text {hi }}$ monocyte recruitment to the blood. Although Stat $2^{-/-}$mice had lower steady-state levels of Ly6 $\mathrm{C}^{\text {hi }}$ monocytes present in the blood, they were able to mobilize Ly6C ${ }^{\text {hi }}$ monocytes from the bone marrow to the blood to the same extent as WT mice (Fig 6A). CCL2 injection in the lung has been reported to recruit $L y 6 C^{\text {hi }}$ monocytes (48), however we were unable to reproduce these results. This is in agreement with work suggesting that CCL2 is required to recruit Ly6 $\mathrm{C}^{\text {hi }}$ monocytes from the bone marrow to the blood, however it is not required for recruitment from the blood to the tissues, suggesting that other signals mediate tissue entry from the blood (1-3).

In a separate approach, we generated mixed bone marrow chimeras by reconstituting WT (CD45.1) mice with a 50:50 mix of WT (CD45.1) and Stat2-- (CD45.2) bone marrow. In these mice, IFN signaling in both WT stromal cells and WT hematopoietic cells can induce CCL2 and CCL7 production. Following infection, mixed bone marrow chimeras show Ly6 $\mathrm{C}^{\text {hi }}$ monocyte as well as neutrophil recruitment to the lung following H5N1 infection (Fig 6B). Approximately equal ratios of WT and Stat $2^{-/}$Ly6 $\mathrm{C}^{\text {hi }}$ monocytes are present at day 2 and day 3 post-infection (Fig 6C). This suggests that IFN signaling in monocytes is not directly required for the recruitment of Ly6C $C^{\text {hi }}$ monocytes to the lung, but likely is required to induce chemokine production that subsequently leads to their recruitment.

\section{IFN-signaling is required for $L y 6 C^{\text {hi }}$ monocyte maturation to Fas $L^{+} M C$ phenotype}

In order to determine the importance of IFN signaling for the maturation of Ly6C ${ }^{\text {hi }}$ monocytes into a FasL $^{+}$MC phenotype, we infected Stat2 ${ }^{-/}$mice with H5N1 and examined surface marker expression. We found that $\mathrm{Ly} 6 \mathrm{C}^{\mathrm{hi}}$ monocytes present in Stat2-/ mice fail to acquire a Fas $\mathrm{L}^{+} \mathrm{MC}$ phenotype - they express lower levels of Ly6C, do not upregulate CD11c, and express lower levels of MHCII. Paradoxically, despite being defective in mounting an IFN-dependent response, they also express lower levels of influenza M2 
protein. Importantly they also fail to upregulate FasL expression (Fig 7A). Because H5N1-infected Stat2-mice fail to induce CCL2 and CCL7 and show only low levels of monocyte recruitment (35), it is unclear whether this represents the same population of cells present in WT mice. In order to determine if Stat $2^{-/-}$ monocytes could mature in the presence of chemokines, we examined the phenotype of these cells in mixed bone marrow chimeras. Although Stat $2^{-/}$Ly $6 \mathrm{C}^{\text {hi }}$ monocytes could be recruited to the lung following H5N1 infection, they did not acquire a FasL ${ }^{+} \mathrm{MC}$ phenotype - they expressed lower levels of Ly6C and CD11c, lower levels of influenza M2, and failed to upregulate FasL (Fig 7B). Importantly no difference in expression of these markers was present in the absence of infection in Ly6 ${ }^{\text {hi }}$ monocytes, or in the absence or presence of infection in neutrophils. Ly6 $\mathrm{C}^{\text {hi }}$ monocytes differentiate into Ly6Clo monocytes, which are involved in tissue repair (10-12). Importantly, while Stat2-- monocytes did not acquire an activated FasL ${ }^{+} \mathrm{MC}^{-1}$ phenotype, they accounted for the majority of Ly6C $C^{10}$ monocytes by day 3 post-infection in mixed bone marrow chimeras (Fig 6C and 7C). The data suggest that upon recruitment to the lungs, monocytes acquire a FasL ${ }^{+} \mathrm{MC}$ phenotype upon exposure to IFN, or differentiate into Ly6 $\mathrm{C}^{\mathrm{lo}}$ monocytes in the absence of IFN signaling.

\section{Discussion}

H5N1 infection is associated with monocytes, monocyte infection, and chemokines

H5N1 infection in humans is characterized by fever, respiratory symptoms, leukopenia, and rapid progression to pneumonia, followed by acute respiratory distress syndrome (ARDS) and multiple organ dysfunction. Lung pathology is characterized by extensive infiltration, diffuse alveolar damage and hyaline membrane formation characteristic of ARDS. The virus has been reported to disseminate systemically in some cases, however most patients die of respiratory failure. A distinctive feature of H5N1 infection is high serum cytokine and chemokine levels referred to as "cytokine storm," which has been suggested to contribute to the severity of disease $(42,43)$. Consistent with chemokine levels, infiltrate primarily composed of monocytes, macrophages, and neutrophils is observed in the lung (49-51). The severe infection, systemic spread, and cytokine phenotypes are also recapitulated in mouse models of H5N1 (52). Here we show that very early following H5N1 infection high numbers of Ly6 $\mathrm{C}^{\text {hi }}$ monocytes are found in the lungs. Many previous studies have identified infiltrating cells as macrophages by either histology or flow cytometry, but given the extent of influx and overlapping marker expression, these cells are likely MCs $(22,36,37,53)$. In addition, tissue-resident alveolar macrophage populations are rapidly depleted during influenza infection (54). Excessive monocyte infiltration has also been reported in SARS-CoV-2 (17-19). CCL2 and CCL7 production in our study correlates with the influx of $L y 6 C^{\text {hi }}$ monocytes, and previous studies have identified Ly6 $\mathrm{C}^{\text {hi }}$ monocytes as the primary producers of these chemokines $(22,36)$. Human histological sections also confirm monocyte/macrophage chemokine expression (55). Studies have also linked CCL2 and CCL7 production to infiltrating monocytes during SARS-CoV-1 (17) and SARS-CoV-2 infection (56). In addition, we find extensive virus infection of Ly6 $\mathrm{C}^{\text {hi }}$ monocytes during H5N1 infection. Virus infection has been observed during H5N1 infection primarily in epithelial cells and monocytes/macrophages $(49,55)$ and in monocytes during H7N9 infection (57), and productive macrophage replication has been suggested to be a unique feature of these viruses $(58)$. We and others $(22,44)$ have observed monocyte infection with H1N1 strains, however in our experiments infection occurs at later time points in lower numbers of cells compared to H5N1 infection. Infection of macrophage subsets has also been reported in SARS-CoV-2 infection (https://doi.org/10.1101/2020.03.27.20045427). Whether infection and/or productive replication in monocytes are important determinants of $\mathrm{H} 5 \mathrm{~N} 1$ pathogenesis requires further investigation. Importantly, we also show that monocytes are the primary IFN producing and responding cells during infection; a finding also supported in vaccinia virus infection and lupus models $(59,60)$.

\section{Monocytes, FasL, TRAIL contribute to pathogenesis}

We show that monocytes contribute to pathogenesis during $\mathrm{H} 5 \mathrm{~N} 1$ infection, as $\mathrm{Ccr} 2^{-/}$mice are protected from mortality. Although $\mathrm{Ccr}^{--}$mice show delayed viral clearance and decreased T cell priming $(20,21)$, they are protected from excessive lung damage and lethal influenza infection in most studies $(20,22,23)$. Treatment with CCR2 depleting antibody also protects from SARS-CoV-1 lethality (17). TRAIL expression by MCs referred to as exudate macrophages contributes to lethal influenza virus infection (23). In addition, higher IFN levels in some mouse strains leads to Ly6 $\mathrm{C}^{\text {hi }}$ monocyte influx and TRAIL expression $(26,38)$. TRAIL neutralization protects from lung damage and death in both cases. Here we found FasL rather than TRAIL expression on Ly6C ${ }^{\text {hi }}$ monocytes. Expression of FasL during influenza virus infection has also been reported in other studies, and neutralization or genetic mutation protects from lethal infection $(45,46)$. We 
show MC expression of FasL, however FasL is also known to regulate T cell apoptosis during influenza virus infection (61), therefore it is possible that the effect of FasL on survival is mediated by cells other than monocytes. However, in the case of TRAIL-mediated tissue damage, T cell deficiency did not affect disease outcome (26). The reasons for differential expression of TRAIL and FasL are unknown but may be due variation in mouse or viral strains. Interestingly, neutralization of TRAIL or FasL during in vitro replication of influenza virus in epithelial cells leads to decreased viral titers, implying that the virus modulates cell death pathways to enhance replication (62). During SARS-CoV-1 infection, Ly6C ${ }^{\text {hi }}$ monocytes also express FasL in an IFN-dependent manner (17), however TNF- $\alpha$ rather than FasL contributes to pathogenesis in this case, highlighting that MCs can mediate pathogenic effects through various mechanisms. Importantly, monocytes are not universally pathogenic; they are protective during RSV, HSV, and WNV infection (48, 63).

IFN not required for recruitment, differentiation to $L y 6 C^{\prime o}$

A number of studies have reported loss of Ly6 $\mathrm{C}^{\text {hi }}$ monocyte recruitment in Ifnar $1^{-/}$mice following infection with influenza $(22,35,36)$, SARS-CoV-1 (17), and SARS-CoV-2 (19), however we show that IFN signaling in monocytes is not directly required for recruitment. In contrast, another report showed lower levels of Ly6C $^{\text {hi }}$ and higher levels of Ly6C ${ }^{l o}$ monocytes in WT: Ifnar1-- mixed bone marrow chimeras (64). However, monocyte recruitment was examined at day 7 in this study, whereas we found approximately equal ratios of WT and Stat $2^{-/}$Ly6 $\mathrm{C}^{\text {hi }}$ monocytes in mixed bone marrow chimeras at both day 2 and 3 post-infection. Given that we found an absence of IFN-signaling was associated with increased Ly6C ${ }^{l o}$ monocytes at day 3 , it is likely that many of the recruited Ifnar $1^{-1}$ Ly6 $C^{\text {hi }}$ cells had converted to Ly6Clo cells by day 7 . A number

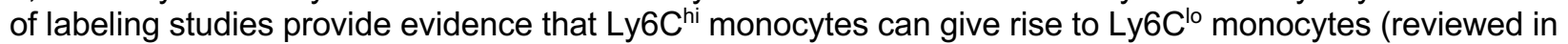
(65)). However Ly6C ${ }^{\text {hi }}$ but not Ly6C ${ }^{10}$ monocyte production is effected by the absence of the transcription factors IRF8 and KLF4 $(8,9)$, suggesting that alternate developmental pathways may exist. In addition, mice deficient for the transcription factor Nur77 lack Ly6C ${ }^{10}$ monocytes specifically (66). The relative importance of these proposed developmental pathways is unknown and is likely to vary under pathologic conditions. In a mouse model of pristane-induced inflammation, IFN-signaling stimulated the production of chemokines that recruited Ly6 $\mathrm{C}^{\text {hi }}$ monocytes to the peritoneum via CCR2. Ifnar/- mice had high levels of Ly6 $\mathrm{C}^{10}$ monocytes consistent with our findings; labeling experiments supported a differentiation of Ly6 $\mathrm{C}^{10}$ cells from Ly6C ${ }^{\text {hi }}$ precursors (67). Regardless of whether Ly6C ${ }^{10}$ monocytes differentiate from Ly6C ${ }^{\text {hi }}$ precursors, or develop independently, type I IFN signaling appears to be important for controlling the balance between the subsets. Ly6C ${ }^{10}$ monocytes are generally thought to differentiate into resident macrophages that promote wound healing and resolution of inflammation. They produce only low levels of proinflammatory cytokines, and higher levels of anti-inflammatory factors including IL-1RA, IL10R, and ApoA/E, and CXCL16 (68). Ly6C ${ }^{10}$ monocytes patrol the vasculature and remove damaged cells $(68,69)$. They also cross-present antigens derived from apoptotic cells, and promote tolerogenic responses through expression of PDL1 (70). How these cells contribute to influenza virus infection remains to be investigated.

\section{IFN required for monocyte maturation to MCs}

Stat $^{-1-}$ Ly6 $\mathrm{C}^{\text {hi }}$ monocytes fail to differentiate into FasL ${ }^{+} \mathrm{MCs}$, indicating that IFN signaling regulates the maturation of Ly6C ${ }^{\text {hi }}$ monocytes into MCs. Consistent with this, monocytes in human blood have been reported to be infected with a number of viruses, and differentiate into MCs that express dendritic cell markers in association with type I IFN production and ISG upregulation $(17,71,72)$. IFN treatment has also been reported to lead to maturation of human monocytes into TRAIL ${ }^{\mathrm{MCs}}$, which induce IL-15 production and promote $T$ cell responses (73). The Aryl hydrocarbon receptor (AhR) and IRF4 have been reported to be involved in differentiation of Ly6C hi monocytes into MCs in both human and mouse experiments (74). Interestingly, we observed much lower levels of Ly6C ${ }^{\text {hi }}$ monocyte infection in Stat $2^{-/}$cells, implying that the differentiation to a MC phenotype in some way influences susceptibility to infection, an observation that is currently under investigation. Important roles for Ly6 $C^{\text {hi }}$ monocyte IFN signaling have also been defined in other infection models. Ly6 $\mathrm{C}^{\mathrm{hi}}$ monocytes control CD8+T cell and NK cell immunity through IFN-induced IL-18 and IL-15 production in response to a variety of bacterial, viral, and parasite infections (75). During HSV-2 infection, IFN signaling in inflammatory monocytes leads to IL-18 production, which subsequently activates NK cells that are critical for clearance of infection (76). In a Candida infection model, type I IFNdependent IL-15 production by inflammatory monocytes in the spleen is critical for the activation of NK cells and neutrophils (77). Thus while IFN appears to have important effects on the maturation of Ly6C ${ }^{\text {hi }}$ monocytes into MCs, the resulting effects are protective in many cases, but pathogenic in some cases. 
Model

Altogether the data suggest a model for how type I IFNs contribute to H5N1 pathogenesis (Fig 8). Upon initial infection, influenza virus is detected by both epithelial and hematopoietic cells resulting in an initial wave of IFN- $\alpha / \beta$ and $\lambda$ production. pDCs and alveolar macrophages have been defined as the primary IFNproducing cells at early time points following respiratory virus infection (78). This IFN is detected by resident Ly6C $^{\text {hi }}$ monocytes, which signal through IFNAR1/2 to induce the production of ISGs including CCL2 and CCL7 - leading to an influx of Ly6 $\mathrm{C}^{\text {hi }}$ monocytes from the bone marrow to the blood. Additional factors control the recruitment from the blood to the lung. Recruitment of these monocytes is not directly dependent on IFN, but is dependent on IFN-induced chemokine production. Once recruited to the lung Ly6C ${ }^{\text {hi }}$ monocytes mature into various $\mathrm{MC}$ effector populations dependent on the level of IFN. In the absence of IFN-signaling Stat2 ${ }^{-/}$cells become Ly6C ${ }^{\circ}$, a phenotype associated with tissue repair. Intermediate levels of IFN likely facilitate beneficial outcomes including T cell priming and viral clearance. In the case of H5N1 infection, Ly6C ${ }^{\text {hi }}$ monocytes become infected, produce high levels of IFN- $\beta$ and CCL2/7 - leading to excess influx, and mature into MCs that expresses CD11c, MHC Class II, and FasL facilitating tissue destruction. 

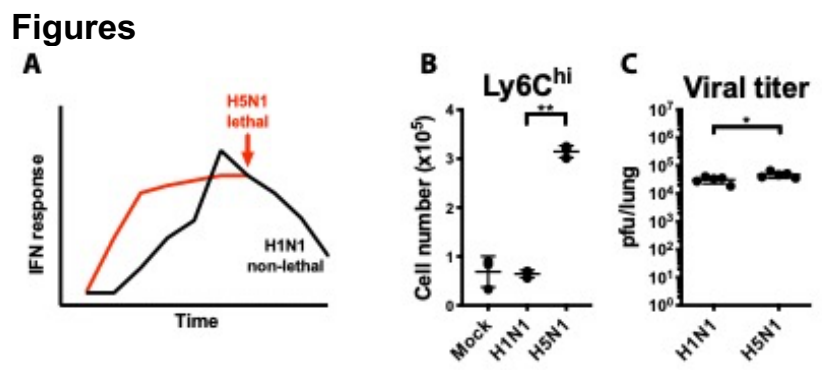

$\mathbf{E}$

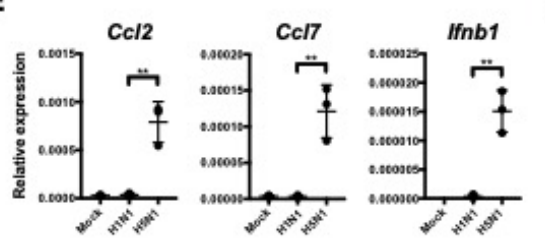

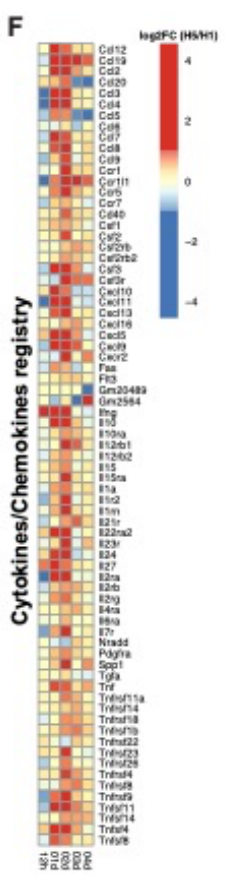

D

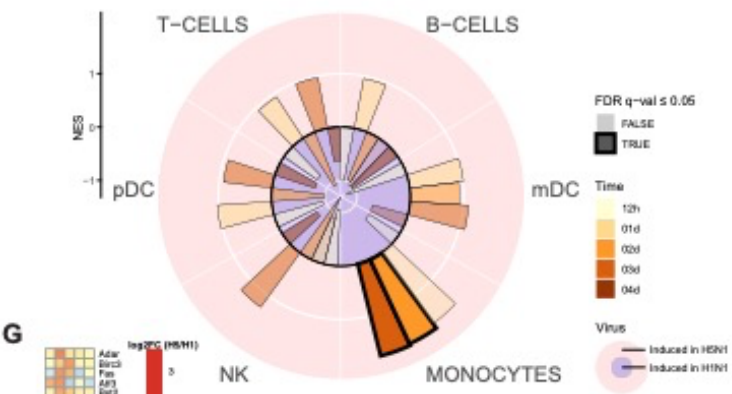

二-iascad nitivit

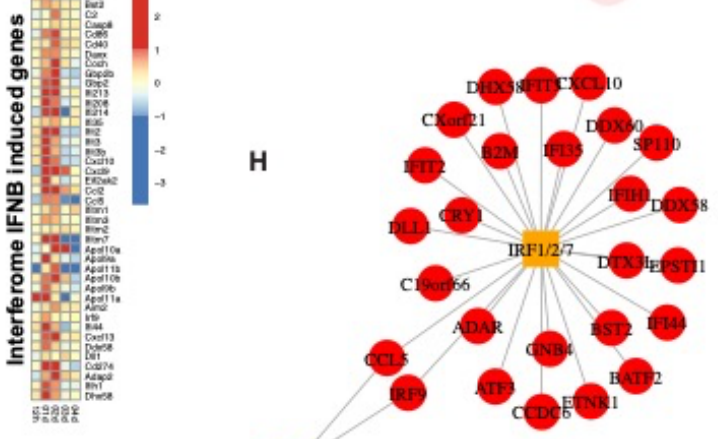

STAT3/SA

Lorem ipsum

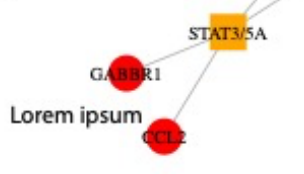

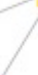

Figure 1. Monocytes are associated with H5N1 infection

(A) IFN response and pathogenesis of $\mathrm{H} 1 \mathrm{~N} 1$ and $\mathrm{H} 5 \mathrm{~N} 1$ at the doses used in this study

(B) Mice were infected with $10^{2}$ pfu of $\mathrm{H} 1 \mathrm{~N} 1$ or H5N1 and Ly6C ${ }^{\text {hi }}$ monocyte (Ly6C ${ }^{\text {hi }}$ CD11 b Ly6G $^{-}$) infiltration and was determined by flow cytometry at day 2 post-infection $(n=3)$.

(C) Mice were infected as in $B$ and viral titer was determined by plaque assay at day 3 post-infection. Data are representative of 3 experiments for $B$ and 2 experiments for $C$. ${ }^{*} p<0.05^{\star *} p<0.01$

(D) Gene Set Enrichment analysis was performed to assess the enrichment of transcriptomic markers of immune cells (B, T, NK, Monocytes, mDC, pDC; (40) among genes differentially expressed between H5N1and H1N1-infected mice in lung tissue. The radial plot presents the Normalized enrichment score (NES) of each set of markers (quadrants) for different timepoints investigated after infection (bars). A NES > 0 correspond to enrichment of cell markers among genes induced in H5N1 compared to H1N1 while a NES $<0$ corresponds to the enrichment of cell markers among genes repressed in H5N1 compared to H1N1. A permutation test was performed to assess the significance of each enrichment; opaque bars correspond to enrichment with a false discovery rate (FDR) below 0.05 .

(E) Mice were infected with $10^{2}$ pfu of H1N1 or H5N1 and Ly6C ${ }^{\text {hi }}$ monocyte (Ly6C ${ }^{\text {hi }}$ CD11 $b^{+}$Ly6G-) infiltration and chemokine induction was determined by qPCR at day 2 post-infection $(n=3)$. Data are representative of 3 experiments. ${ }^{* *} p<0.01$

(F) Heatmap showing the log2 fold-change (log2FC) of genes coding for cytokines/chemokines between H5N1- and H1N1-infected mice in lung tissue. A blue-white-red color gradient depicts the gene the most repressed to the gene the most induced in H5N1-infected mice compared to H1N1-infected mice.

(G) Gene Set Enrichment Analysis (GSEA) was performed to assess the enrichment (i.e. overlap) of interferon-stimulated genes (IFN- $\alpha, \beta, \lambda$, and $\gamma$ induced genes; (80)) among genes differentially expressed between H5N1- and H1N1-infected mice in lung tissue. This analysis revealed a significant enrichment of IFN- $\beta$-induced genes in the lungs of H5N1-infected mice on day 2. The heatmap shows the log2FC of 
bioRxiv preprint doi: https://doi.org/10.1101/2022.01.10.475751; this version posted January 11 2022. The copyright holder for this preprint (which was not certified by peer review) is the author/funder, who has granted bioRxiv a license to display the preprint in perpetuity. It is made available under aCC-BY-ND 4.0 International license.

genes contributing to the IFN- $\beta$-induced gene enrichment in $\mathrm{H} 5 \mathrm{~N} 1$-infected mice compared to H1N1infected mice.

(H) GSEA was used to identify transcription factors (TF) upstream of IFN- $\beta$-induced genes in the lungs of H5N1-infected mice on day 2. In the network, TF (scare) and their targets (circle) are linked (edge) if there is a putative binding sites in the 2000 bases around the TSS of the target genes or that the genes was differentially regulated in TF-overexpression or knock-out experiments.

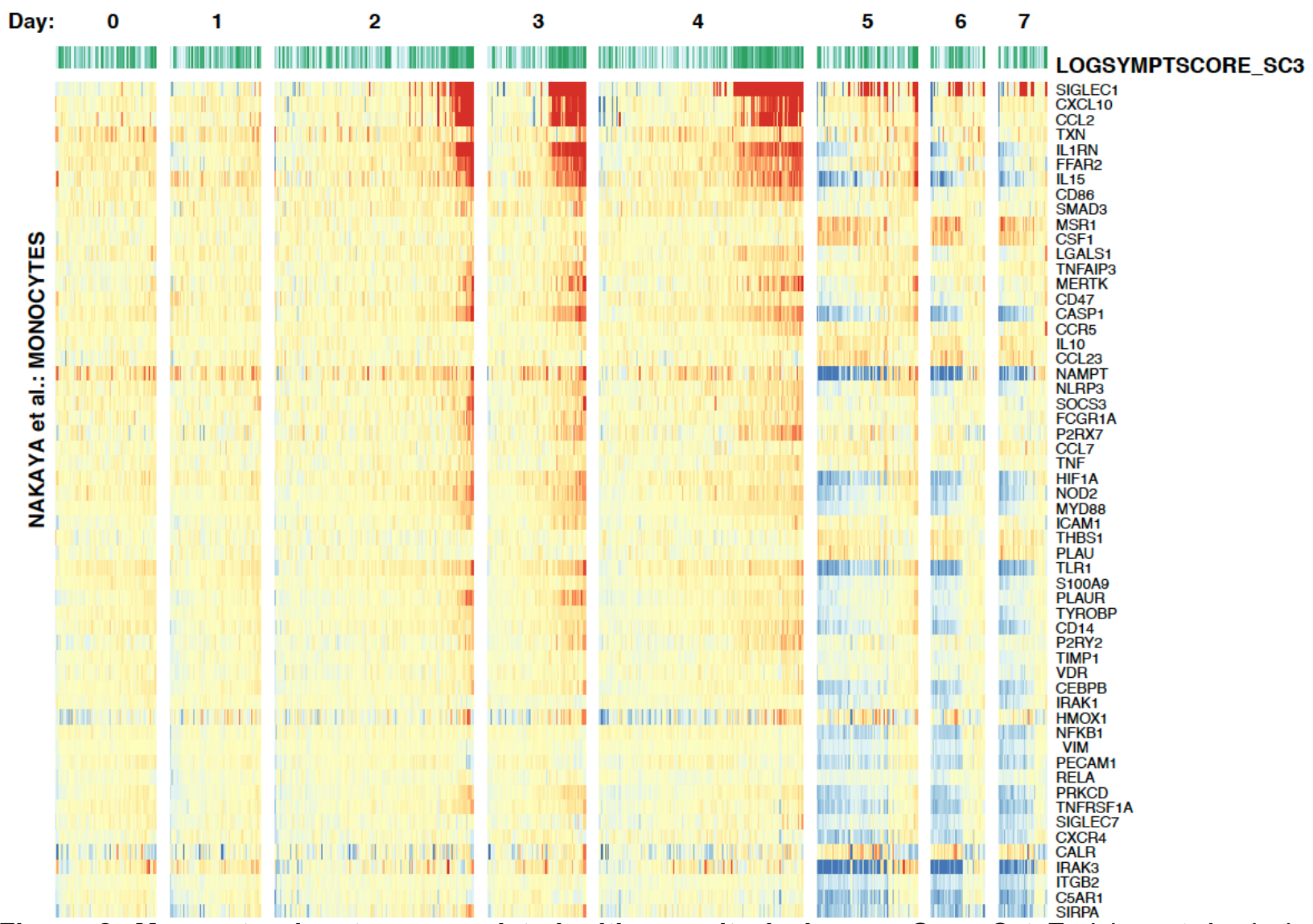

Figure 2. Monocyte signature associated with severity in human Gene Set Enrichment Analysis (GSEA) was performed to assess the enrichment (i.e. overlap) of transcriptomic markers of immune cells $(\mathrm{B}, \mathrm{T}, \mathrm{NK}$, Monocytes, mDC, pDC) among genes expressed in blood of healthy adults challenged with H1N1 and correlated with severity of symptoms (LOGSYMTOMSCORE; (79)). A significant enrichment of monocyte signature was observed on days 2, 3 and 4 . The heatmap shows the expression of transcriptomic markers of monocytes contributing to this enrichment. A blue-white-red color gradient represents downregulation to up-regulation of the gene-expression scaled across samples (z-score). 

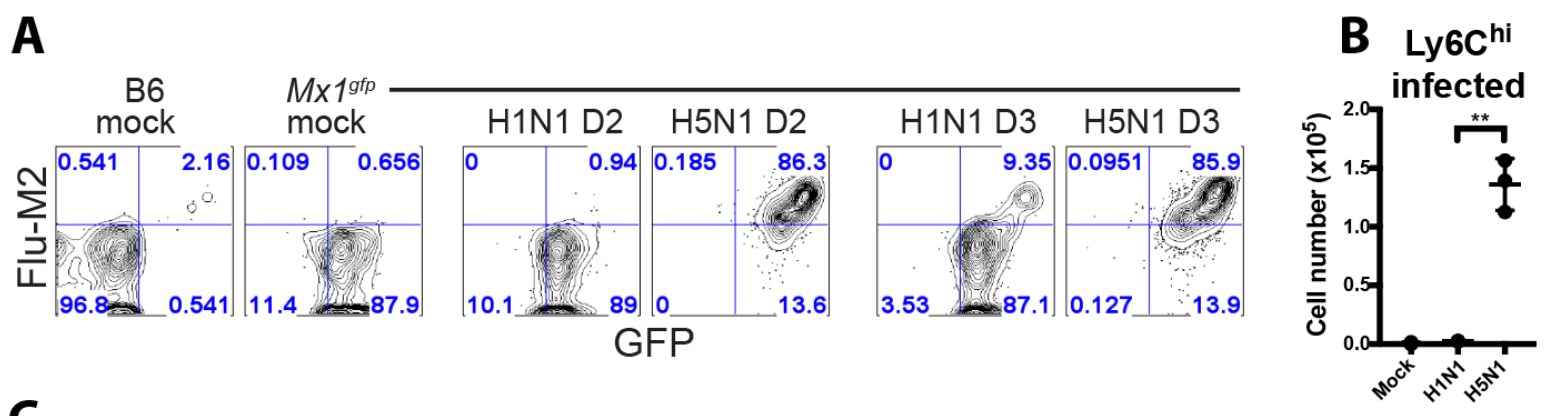

C
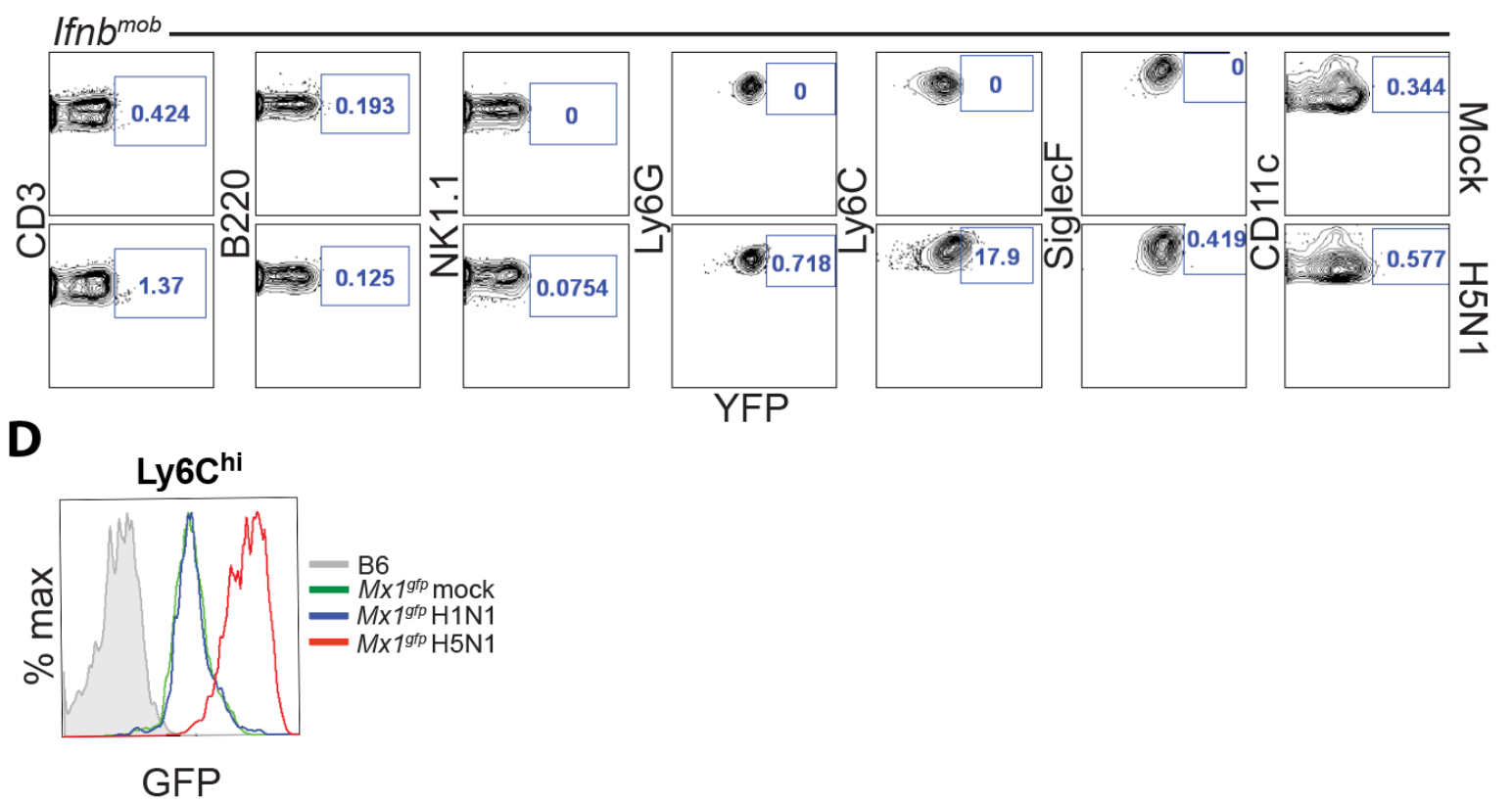

Figure 3. Monocytes become infected and produce IFN- $\beta$ and ISGs

454 (A) $M \times 1^{\text {gfp }}$ mice were infected with $10^{2}$ pfu of H1N1 or H5N1 and surface Flu-M2 expression on Ly6C ${ }^{\text {hi }}$ monocytes was determined by flow cytometry at day 2 and 3 post-infection.

(B) Quantification of $A(n=3)$. Data are representative of 3 experiments. ${ }^{* *} p<0.01$.

(C) Ifnb $b^{m o b}$ mice were infected with $10^{2}$ pfu of H5N1 and YFP expression was measured by flow cytometry at day 3 post-infection $(n=2)$. Data are representative of 3 experiments. (D) $M \times 1^{\text {gfp }}$ mice were infected with $10^{2}$ pfu of H1N1 or H5N1 and GFP expression was measured in Ly6C ${ }^{\text {hi }}$ monocytes. Data are representative of 3 experiments. 
A
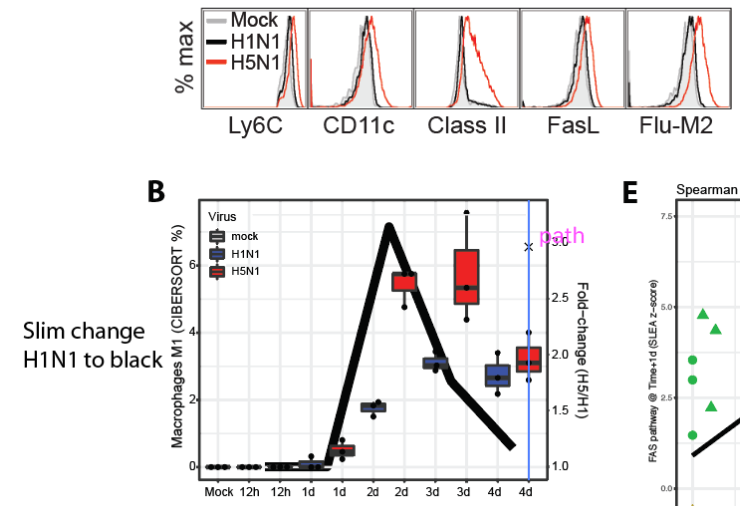

C

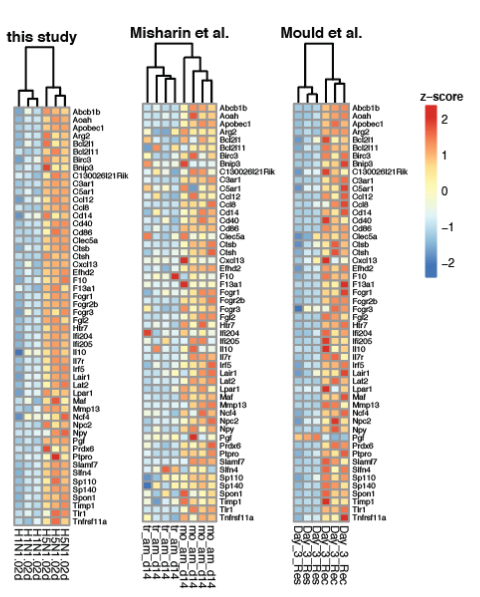

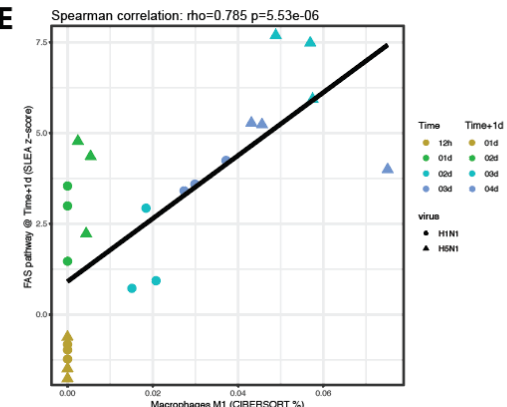

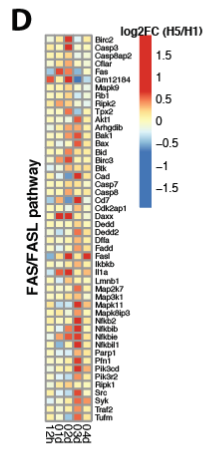

Figure 4. Monocytes mature into $M 1 / F a s L+M C$ phenotype during severe infection

(A) Mice were infected with $10^{2}$ pfu of $\mathrm{H} 1 \mathrm{~N} 1$ or H5N1 and expression of the indicated markers on Ly6C ${ }^{\text {hi }}$ monocytes was determined at day 2 post-infection $(n=3)$. Data are representative of 3 experiments. ${ }^{* *} \mathrm{p}<0.01$

(B) Cibersort (81) was used to infer cell frequencies based on RNA-Seq data. Boxplot of inferred Macrophage M1 (IL-12 ${ }^{\text {high }}$, IL-23 high, IL-10 low phenotype, polarized by LPS) frequencies in lung of H5N1-, H1N1- and mock-infected mice at different timepoints following infection.

(C) Transcriptomic expression of genes induced in monocyte-derived macrophages compared to tissue resident macrophages in the lung of H5N1- and H1N1-infected mice. GSEA enrichment analysis was used to test for enrichment of transcriptomic markers of monocyte-derived macrophages and of tissue resident macrophages $(82,83)$. A significant enrichment of monocyte-derived macrophage markers among genes induced in H5N1-vs. H1N1-infected mice. Genes contributing to the enrichment (i.e. overlapping monocytederived macrophages and induced in H5N1 infected mice) are shown in the heatmaps. A blue-white-red color gradient represents down-regulation to up-regulation of the gene-expression scaled across samples (z-score).

(D) Gene Set Enrichment Analysis (GSEA) was performed to assess the enrichment (i.e. overlap) of transcriptomic markers of cell death pathways (Apoptosis, Pyroptosis, Necrosis) among genes differentially expressed between H5N1- and H1N1-infected mice in lung tissue. This analysis revealed a significant enrichment of Fas-mediated cell death in the lung of H5N1-infected mice at day 3 . The heatmap shows the log2FC of genes contributing to the Fas pathway enrichment in H5N1-infected mice compared to H1N1infected mice.

(E) Scatter plot showing the levels of Fas pathway (summarized using the SLEA z-score method) in the lung of H5N1-, H1N1- and mock-infected mice as a function of the inferred frequency of M1 macrophage (inferred from Cibersort) the preceding day. A Spearman correlation and t-test was used to statistically assess the correlation. 

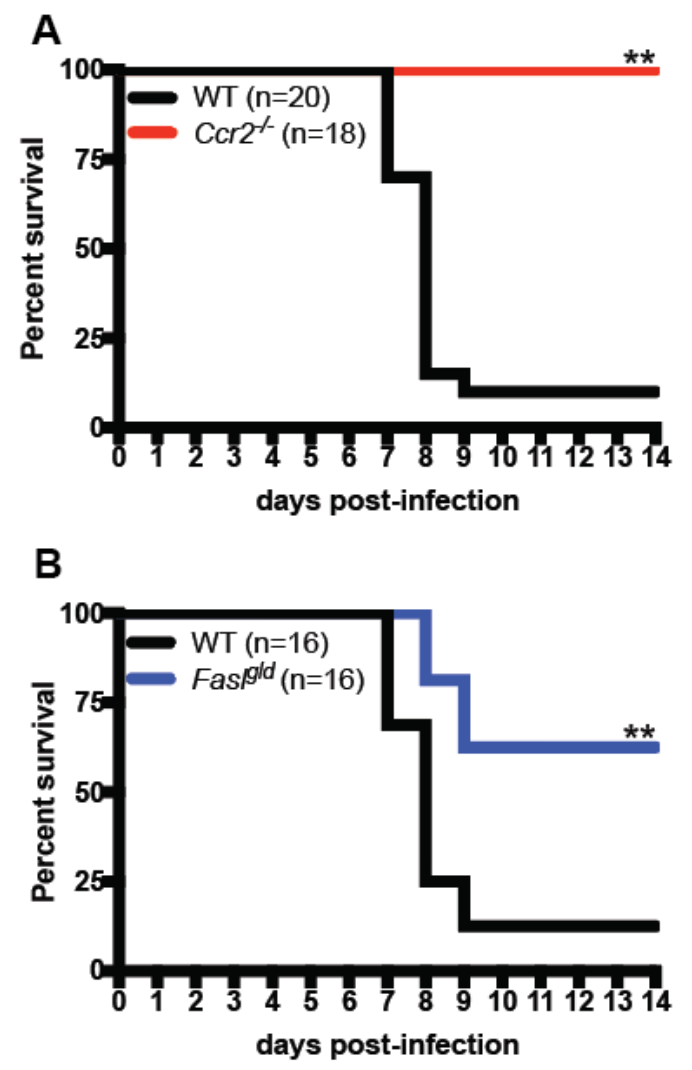

Figure 5. Monocytes contribute to lethality during severe infection.

(A) WT and $\mathrm{Ccr}^{-/-}$mice were infected with 10 pfu of H5N1 and survival was measured.

(B) WT and Fas ${ }^{\text {gld }}$ mice were infected as in A and survival was measured. Data show combined results of 
A
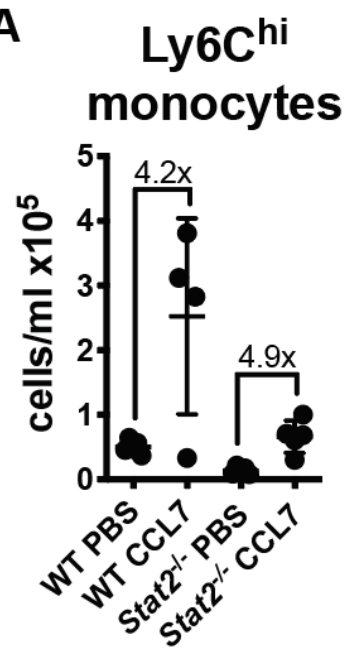

B
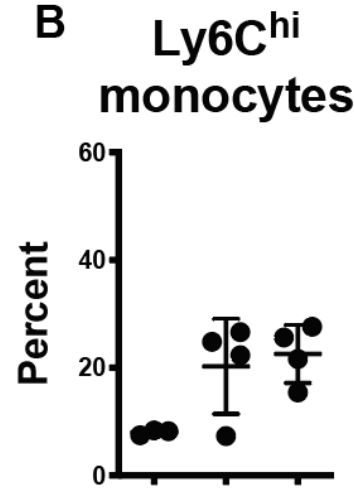

C

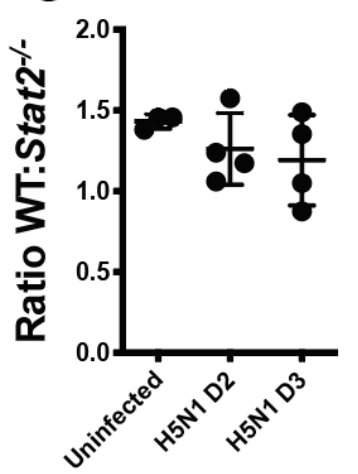

Ly6Clo monocytes Neutrophils
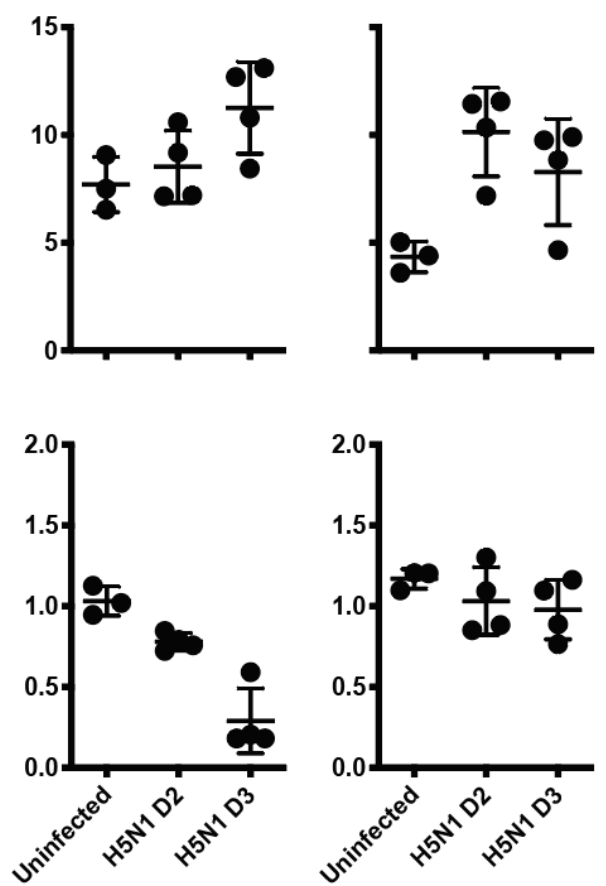

Figure 6. IFN-signaling is not directly required for monocyte recruitment.

(A) WT and Stat2-- mice were injected IV with CCL7 and Ly6C ${ }^{\text {hi }}$ monocytes in the blood were measured by flow cytometry at $30 \mathrm{~min}(\mathrm{n}=4)$. (B) WT:Stat2-- $\rightarrow$ WT 50:50 mixed bone marrow chimeras were infected with $10^{2}$ pfu of $\mathrm{H} 5 \mathrm{~N} 1$ and Ly6 $\mathrm{C}^{\mathrm{hi}}$ and Ly6 $\mathrm{C}^{\mathrm{lo}}$ monocyte, and neutrophil influx was measured by flow cytometry at day 2 and 3 post-infection $(n=4)$. Data are representative of 2 experiments. 
A

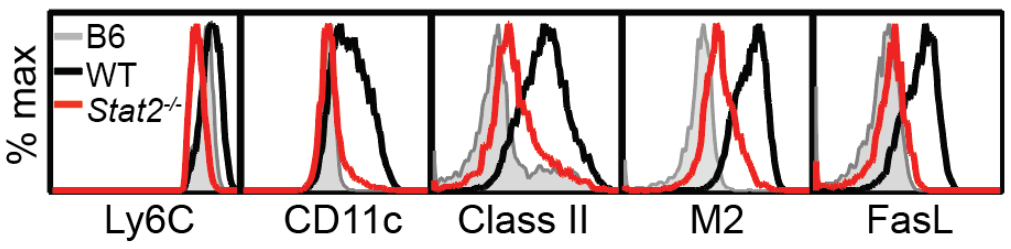

B

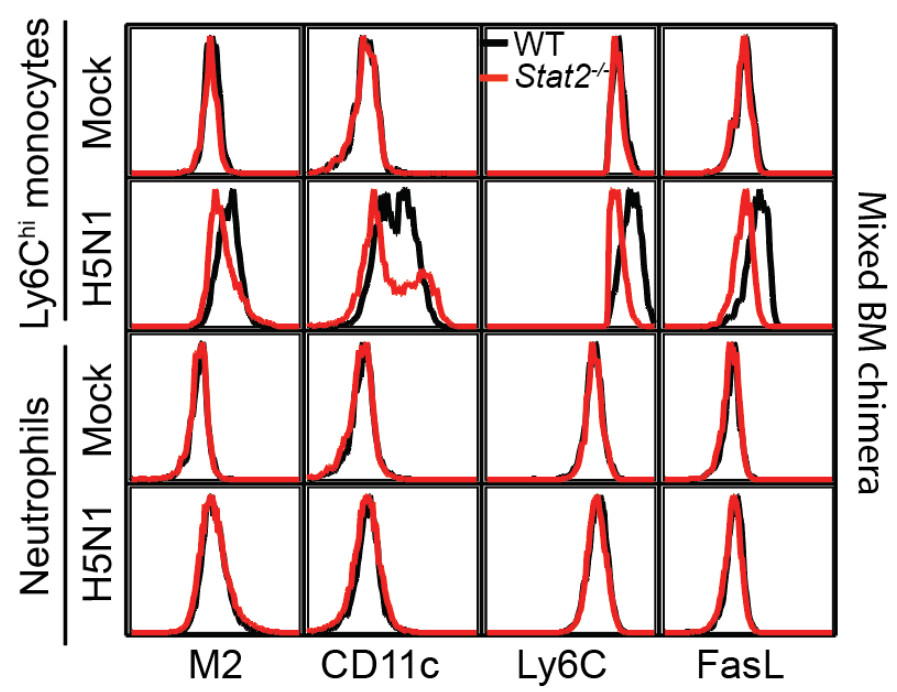

C

Ly6C hi Ly6C $C^{10}$

monocytes monocytes Neutrophils

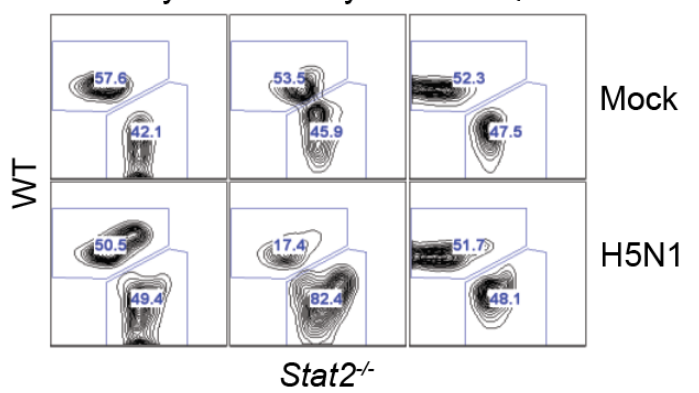

511
Figure 7. IFN signaling is required for maturation to Fas $L^{+}$MCs. (A) WT and Stat $2^{-/-}$mice were infected with $10^{2}$ pfu of $\mathrm{H} 5 \mathrm{~N} 1$ and expression of the indicated markers on Ly6C $\mathrm{C}^{\text {hi }}$ monocytes was determined at day 2 post-infection $(n=3)$. (B) WT:Stat2 ${ }^{--} \rightarrow$ WT 50:50 mixed bone marrow chimeras were infected with $10^{2}$ pfu of H5N1 and expression of the indicated markers on Ly6 $\mathrm{C}^{\text {hi }}$ monocytes and neutrophils was determined by flow cytometry $(n=4)$. Data are representative of 3 experiments for $A$ and 2 for $B$. 
CHEMOKINES

IFN-dependent

\section{RECRUITMENT}

IFN-independent

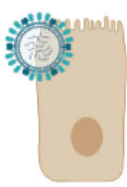

Epithelial cell

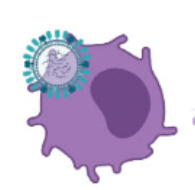

pDC

\section{IFN- $\beta$}

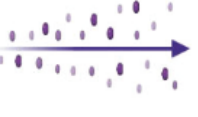

M

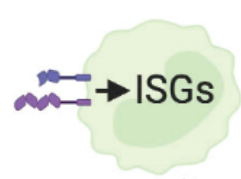

Ly6 $\mathrm{C}^{\text {hi }}$

monocyte

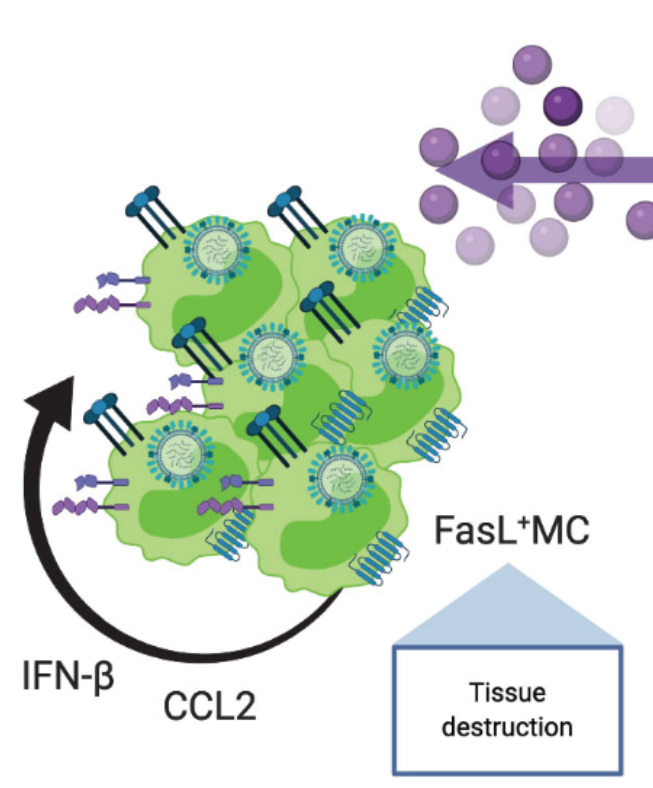

MATURATION

IFN-dependent

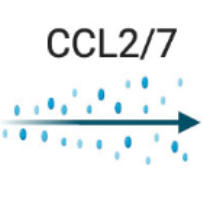

( 
A

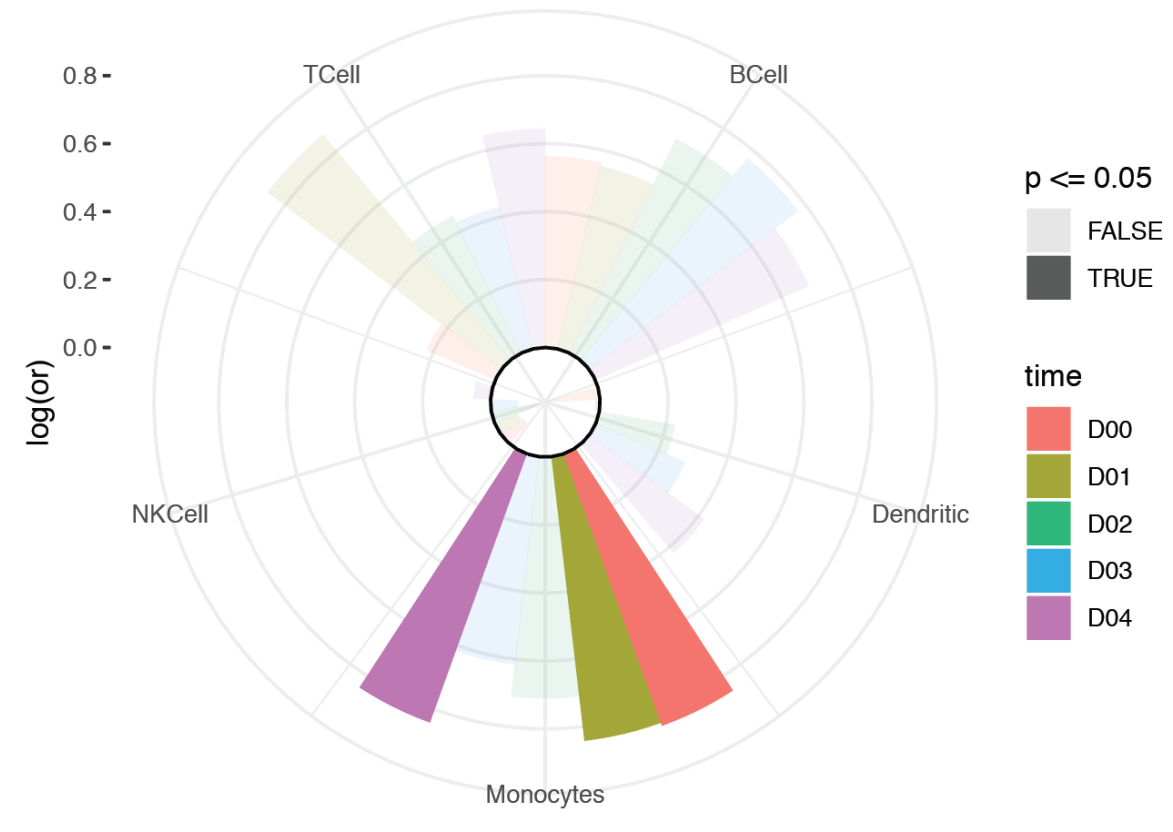

C

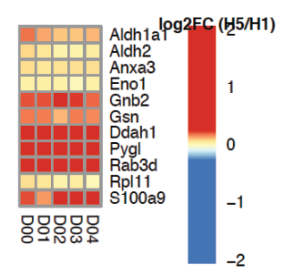

B

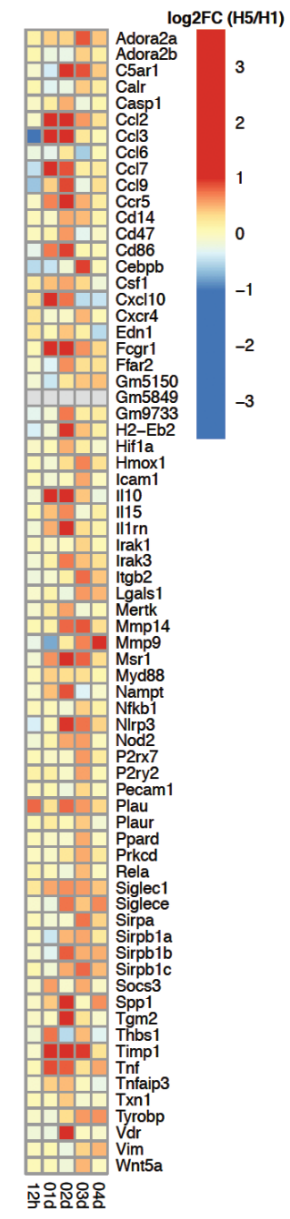

Figure S1. Proteic markers of monocytes are preferentially induced following H5N1 infection

(A) Fisher's exact test was used to assess statistically the overlap between protein markers of immune cells (B, T, NK, Monocytes, DC) among proteins differentially expressed between H5N1- and H1N1-infected mice in lung tissue. The radial plot presents the log odd ratio (log(or)) of each set of markers (quadrants) for different timepoints investigated after infection (bars). A log odd ratio $>0$ correspond to overlap of cell markers among proteins induced in $\mathrm{H} 5 \mathrm{~N} 1$ compared to $\mathrm{H} 1 \mathrm{~N} 1$ while a log odd ratio $<0$ corresponds to the enrichment of cell markers among proteins repressed in H5N1 compared to H1N1. Benjamini-Hochberg correction was used to adjust for false positives. (B) Heatmap showing the log2 fold-change (log2FC) of monocytes transcriptomic markers between $\mathrm{H} 5 \mathrm{~N} 1$ - and $\mathrm{H} 1 \mathrm{~N} 1$-infected mice in lung tissue. A blue-whitered color gradient depicts the gene the most repressed to the gene the most induced in H5N1-infected mice compared to H1N1-infected mice. (C) Heatmap showing the log2 fold-change (log2FC) of monocytes proteomic markers between $\mathrm{H} 5 \mathrm{~N} 1$ - and $\mathrm{H} 1 \mathrm{~N} 1$-infected mice in lung tissue. A blue-white-red color gradient depicts the protein the most repressed to the protein the most induced in H5N1-infected mice compared to H1N1-infected mice. 
A

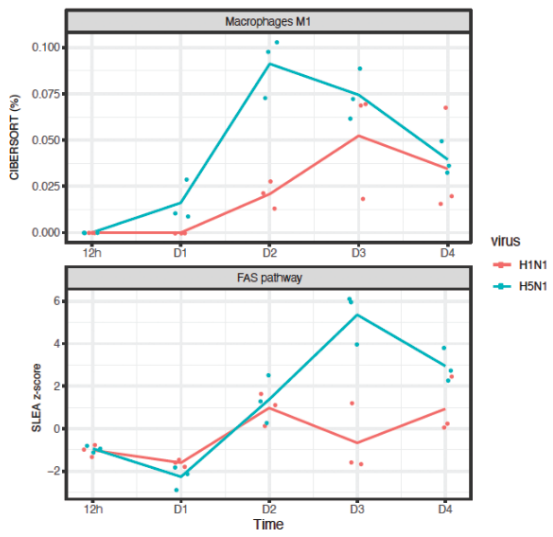

B

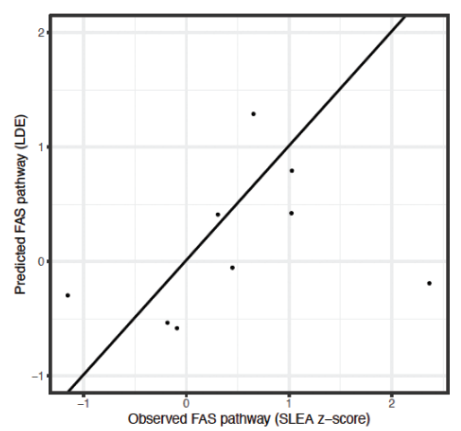

C

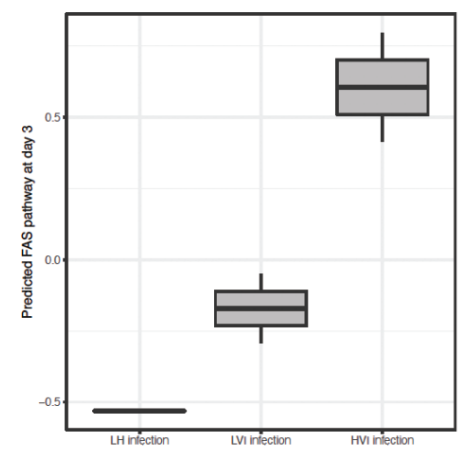

Figure S3. FAS pathway activation by M1 macrophages

(A) Inferred frequency of M1 macrophages (upper panel) and FAS pathway activation (lower panel) as a function of time. M1 macrophages recruitment was maximal two days following infection with H5N1 while FAS pathway was maximum activation was 1 day later (day three following infection with H5N1). (B) Mathematical modeling of FAS pathway at day $d$, was expressed as a linear function of FAS pathway activation at day $\mathrm{d}-1$ and M1 macrophage frequency at day-1. The resulting model was confirmed on a publicly available set of transcriptomic data of mice infected with mock (LH), low virulent H3N2 (LVI) and highly virulent $\mathrm{H} 3 \mathrm{~N} 2$ virus $(\mathrm{HVI})$. The activation of FAS pathway predicted by the mathematical model ( $\mathrm{y}$ axis) is presented as a function of the observed activation of FAS pathway (x-axis). The resulting Spearman correlation was 0.617 and a t-test $p$-value of 0.0857 . (C) Boxplot of the activation of FAS pathway predicted by the mathematical model as a function of $\mathrm{H} 3 \mathrm{~N} 2$ virulence. 


\section{Figure S2 Cell subset frequencies in mice lung following influenza infections} frequency of a subsets (color) in each triplicated samples (bars). 
602

603

604

605

606

607

608

609

610

611

612

613

614

615

616

617

618

619

620

621

622

623

624

\section{Materials and Methods \\ Mice}

Animal studies were approved by the Institutional Animal Care and Use Committee of Icahn School of

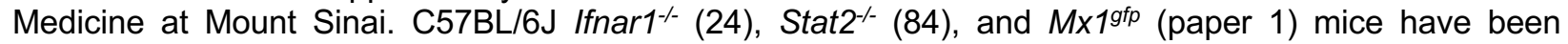
previously described. Ifnb ${ }^{\mathrm{mob}}, \mathrm{Ccr}^{-/}$, and Fas ${ }^{\text {gld }}$ mice on the $\mathrm{C} 57 \mathrm{BL} / 6 \mathrm{~J}$ background were purchased from Jackson. For mixed bone marrow chimeras 6-week old B6.SJL-Ptprc ${ }^{a}$ Pepc $^{\text {b/BoyJ }}$ (CD45.1) female mice were irradiated with 2 doses of 600 rads and injected with a 50:50 mix of WT (CD45.1) and Stat2-/- (CD45.2) bone marrow.

\section{Lung isolations and flow cytometry}

Lungs were digested for $40 \mathrm{~min}$ in $1 \mathrm{mg} / \mathrm{ml}$ collagenase type 4 (Worthington) $5 \%$ FBS in DMEM. Cells were then filtered through a $0.2 \mu \mathrm{m}$ cell strainer and RBCs were lysed. Cells were suspended in $3 \% \mathrm{FBS} 2 \mathrm{mM}$ EDTA in PBS and staining was performed in the presence of $2 \%$ NRS, $2 \%$ Fc block (BD), and fixable viability dye eFluor 450 (ebiosciences). Cells were stained with the following antibodies from BD: Ly6CPerCP-Cy5.5 (AL-21), CD11b-PE (M1/70), Ly6G-V450 (1A8), CD11C-V450 or PE-Cy7 (HL3), CD45.1-FITC (A20), CD45.2-PE-CF594 (104), the following antibodies from eBiosciences: MHC Class II (I-A/I-E) e450 (M5/114.15.2), FasL-APC (MFL3), and from R\&D: CCR2-APC (475301). Influenza M2 (E10) (85) was conjugated to Alexa 647 . Cells were fixed with $2 \%$ formaldehyde after staining and analyzed on an LSRII after gating for FSC/SSC, singlets, and live cells.

Infections and chemokine treatment

Mice were infected with the following viruses at the doses indicated in $20 \mu \mathrm{lPBS}$ : A/PR/8/34 (H1N1) (PR8), A/Viet Nam/1203/04 (H5N1) lacking the multibasic cleavage site (HALo)(39). Viral titer was determined by plaque assay on MDCK cells. Mice were injected IV with $500 \mathrm{ng}$ of CCL7 (47) and blood was harvested 30 min later in EDTA, RBCs were lysed and cells were analyzed by flow cytometry.

\section{qRT-PCR}

Total RNA was extracted from collagenase-digested lung using using EZNA total RNA kit and RNase-free DNase (Omega). RNA was reverse-transcribed using Maxima Reverse Transcriptase and oligo-dT (Thermo). Quantitative RT-PCR was performed on cDNA using LightCycler 480 SYBR Green I Master Mix (Roche) and the primers ttgacccgtaaatctgaagctaat-Cc/2F, tcacagtccgagtcacactagttcac-Cc/2R, ggatctctgccacgcttctg-Ccl7F, tccttctgtagctcttgagattcctc-Ccl7R, cacagccctctccatcaacta-Ifnb1F, catttccgaatgttcgtcct-Ifnb1R, gtaacccgttgaaccccatt-18SF, and ccatccaatcggtagtagcg-18SR on a LightCycler $480 \mathrm{II}$ and expressed as $2^{-\Delta \Delta \mathrm{Ct}}$ relative to $18 \mathrm{~S}$.

\section{RNA-Seq analysis}

Mouse lungs were homogenized in liquid nitrogen. $1 \mathrm{ml}$ Trizol was added per 50-100 $\mathrm{mg}$ of tissue and the homogenate was incubated at room temperature for 5 minutes. $200 \mu \mathrm{l}$ chloroform were added per $\mathrm{ml}$ of Trizol, shaken vigorously for $15-30$ seconds and incubated at room temperature for 15 minutes. Samples were centrifuged for 15 minutes at $4^{\circ} \mathrm{C}$ and 12,000 rcf. The aqueous phase was carefully collected in a separate tube. $500 \mu \mathrm{l}$ isopropanol was added per $1 \mathrm{ml}$ Trizol, inverted to mix and incubated at room temperature for 5-10 minutes. Samples were transferred to Qiagen RNeasy Spin Columns and RNA was isolated according to the manufacturer's instructions.

Libraries were constructed using the Illumina TruSeq Stranded Total RNA Library Prep Kit. Pair-end sequencing of 126 base pair reads was used. Raw reads were trimmed using Trimmomatic (version 0.36) using default value for pair-end sequencing. The trimmed reads were then aligned to the mouse genome (build GRCm38.91) using the STAR aligner (version 2.5.3a). Aligned reads were then counted using HTSeq (version 0.9.1). Differential expressed genes between H5N1- and $\mathrm{H} 1 \mathrm{~N} 1$-infected mice was determined by fitting a generalized linear model with gene counts as dependent variable and the virus strain (H5N1 or $\mathrm{H} 1 \mathrm{~N} 1$ ) as independent variable. A likelihood ratio test and Benjamini-Hoshberg correction, as implemented in the $R$ package edgeR, was used to assess the statistical significance of the differential expression. Gene Set Enrichment Analysis (GSEA) was used to assess the significance of MSigDB (version 6.2), bloodcell markers (PMID: 21743478, 28263321) and macrophages signatures (PMID: 28694385, 28421818), cytokines/chemokines (https://www.immport.org/resources/cytokineRegistry) and interferome (version 2) genesets. Gene were ordered from the most induced after H5N1 infection to the gene the most repressed by $\mathrm{H} 5 \mathrm{~N} 1$ infection compared to $\mathrm{H} 1 \mathrm{~N} 1$ using edgeR $p$-values (i.e. $-\log 10(\mathrm{p}) \times \operatorname{sign}(\log F C)$ ). Default 
parameters were used except the maximum genesets size was increased to 2000 genes and fixed seed for the permutation test equal 101. Cibersort (version 1.03) was used to infer cell subsets frequency based on RNA-Seq data.

CIBERSORT (version 1.03) was used to infer immune cell subset frequencies using default parameters.

\section{Microarray analysis}

The human blood dataset of healthy individuals challenged with H1N1 was previously described in [PMID: 30356117]. Briefly, two independent microarray datasets (DEE3 H1N1, DEE4X H1N1) were combined. Each of these studies are from human viral exposure trials where healthy volunteers were followed for 7-9 days following controlled nasal exposure to H1N1 virus. Subjects enrolled into these viral exposure experiments had to meet several inclusion and exclusion criteria. Among them was an evaluation of preexisting neutralizing antibodies to the viral strain. Any subject with pre-existing antibodies to the viral strain was excluded. All subjects exposed to $\mathrm{H} 1 \mathrm{~N} 1$ influenza received oseltamivir 5 days post-exposure. All subjects provided written consents, and each of the seven trials was reviewed and approved by the appropriate governing IRB. Symptom data and nasal lavage samples were collected from each subject on a repeated basis over the course of 7-9 days. Viral infection was quantified by measuring release of viral particles from nasal passages (viral shedding), as assessed from nasal lavage samples via qualitative viral culture and/or quantitative influenza RT-PCR. Symptom data were collected through self-report on a repeated basis. Symptoms were quantified using a modified Jackson score, which assessed the severity of eight upper respiratory symptoms (runny nose, cough, headache, malaise, myalgia, sneeze, sore throat, and stuffy nose) rated $0-4$, with 4 being most severe. Scores were integrated daily over 5-day windows. Blood was collected and gene expression of peripheral blood was performed 1 day $(24-30 \mathrm{~h})$ prior to exposure, immediately prior to exposure, and at regular intervals following exposure. These peripheral blood samples were gene expression profiled on the Affy Human Genome U133A 2.0 array. Only raw (CEL files) gene expression data that pass QC metrics including those for RNA degradation, scale factors, percent genes present, $\beta$-actin $3^{\prime}$ to $5^{\prime}$ ratio and GAPDH $3^{\prime}$ to $5^{\prime}$ ratio in the Affy Bioconductor package were used for downstream analysis. Normalization via RMA was performed on all expression data across all timepoints. The primary endpoint used for differential expression analysis was the symptom score as continuous variable indicating the log of the maximum 5-day integrated symptom score +1 . Linear regression using the $R$ package LIMMA was used to identify genes correlated to the symptom score. GSEA on the gene list ranked by the LIMMA t-static and using the blood-cell markers (PMID: 21743478, 28263321) as reference database was used to identify immune cell subsets correlated to the symptom score.

\section{Proteomics}

Protein abundance in the lung homogenates was measured using amine-reactive isobaric tags in an untargeted run. Briefly, after lysis and protein digestion, peptides from the lung homogenates were labeled with a Tandem Mass Tag (TMT; Thermo Fisher) and mixed in equal proportions. These mixed samples were analyzed by LC-MS on a Thermo Orbitrap Fusion with extended run times to ensure deep coverage. All samples were prepared in biological triplicate and run in technical duplicate to increase sequencing depth. Peptide searches using MaxQuant and statistical testing using MSstatTMT were performed. Fisher exact test was used to assess enrichment of cell subset markers among proteins differentially expressed between H5N1- and H1N1-infected mice.

\section{Statistical analysis}

A Wilcoxon rank-sum test was used to compare levels between two groups. A Spearman correlation and a Student $t$-test was used to assess the correlation between two continuous variables.

\section{Author contributions}

MBU conceived the project, performed the experiments, generated the figures and wrote the manuscript. AGS supervised the work, provided funding, and critically reviewed the manuscript.

\section{Acknowledgements}

We thank Christian Schindler for originally providing the Stat2-- mice. We are grateful to Richard Cadagan and Osman Lizardo for excellent technical assistance. This work was partially supported by CRIP (Center 
680 for Research on Influenza Pathogenesis), an NIAID funded Center of Excellence for Influenza Research and Surveillance (CEIRS, contract \# HHSN272201400008C to AG-S, RA and MBU).

682

DATA AND SOFTWARE AVAILABILITY

685

Data availability

686

687

688

689

RNA-Seq have been deposited in the NCBI's Gene Expression Omnibus with the accession number GSE98527. Proteomics data have been deposited in MassIVE and will be made available with publication of the article.

Code availability

691

All the source code used to generate the figures part of this manuscript is available at of this study are available from the authors upon request. 


\section{References}

1. Tsou, C. L., W. Peters, Y. Si, S. Slaymaker, A. M. Aslanian, S. P. Weisberg, M. Mack, and I. F. Charo. 2007. Critical roles for CCR2 and MCP-3 in monocyte mobilization from bone marrow and recruitment to inflammatory sites. J Clin Invest 117: 902-909.

2. Jia, T., N. V. Serbina, K. Brandl, M. X. Zhong, I. M. Leiner, I. F. Charo, and E. G. Pamer. 2008. Additive roles for MCP-1 and MCP-3 in CCR2-mediated recruitment of inflammatory monocytes during Listeria monocytogenes infection. J Immunol 180: 6846-6853.

3. Serbina, N. V., and E. G. Pamer. 2006. Monocyte emigration from bone marrow during bacterial infection requires signals mediated by chemokine receptor CCR2. Nat Immunol 7: 311-317.

4. Sunderkotter, C., T. Nikolic, M. J. Dillon, N. Van Rooijen, M. Stehling, D. A. Drevets, and P. J. Leenen. 2004. Subpopulations of mouse blood monocytes differ in maturation stage and inflammatory response. J Immunol 172: 4410-4417.

5. Varol, C., L. Landsman, D. K. Fogg, L. Greenshtein, B. Gildor, R. Margalit, V. Kalchenko, F. Geissmann, and S. Jung. 2007. Monocytes give rise to mucosal, but not splenic, conventional dendritic cells. J Exp Med 204: 171-180.

6. MacDonald, K. P., J. S. Palmer, S. Cronau, E. Seppanen, S. Olver, N. C. Raffelt, R. Kuns, A. R. Pettit, A. Clouston, B. Wainwright, D. Branstetter, J. Smith, R. J. Paxton, D. P. Cerretti, L. Bonham, G. R. Hill, and D. A. Hume. 2010. An antibody against the colonystimulating factor 1 receptor depletes the resident subset of monocytes and tissueand tumor-associated macrophages but does not inhibit inflammation. Blood 116: 3955-3963.

7. Yona, S., K. W. Kim, Y. Wolf, A. Mildner, D. Varol, M. Breker, D. Strauss-Ayali, S. Viukov, M. Guilliams, A. Misharin, D. A. Hume, H. Perlman, B. Malissen, E. Zelzer, and S. Jung. 2013. Fate mapping reveals origins and dynamics of monocytes and tissue macrophages under homeostasis. Immunity 38: 79-91.

8. Alder, J. K., R. W. Georgantas, 3rd, R. L. Hildreth, I. M. Kaplan, S. Morisot, X. Yu, M. McDevitt, and C. I. Civin. 2008. Kruppel-like factor 4 is essential for inflammatory monocyte differentiation in vivo. J Immunol 180: 5645-5652.

9. Kurotaki, D., N. Osato, A. Nishiyama, M. Yamamoto, T. Ban, H. Sato, J. Nakabayashi, M. Umehara, N. Miyake, N. Matsumoto, M. Nakazawa, K. Ozato, and T. Tamura. 2013. Essential role of the IRF8-KLF4 transcription factor cascade in murine monocyte differentiation. Blood 121: 1839-1849.

10. Auffray, C., D. Fogg, M. Garfa, G. Elain, O. Join-Lambert, S. Kayal, S. Sarnacki, A. Cumano, G. Lauvau, and F. Geissmann. 2007. Monitoring of blood vessels and tissues by a population of monocytes with patrolling behavior. Science 317: 666-670.

11. Nahrendorf, M., F. K. Swirski, E. Aikawa, L. Stangenberg, T. Wurdinger, J. L. Figueiredo, P. Libby, R. Weissleder, and M. J. Pittet. 2007. The healing myocardium sequentially mobilizes two monocyte subsets with divergent and complementary functions. $J$ Exp Med 204: 3037-3047.

12. Shechter, R., O. Miller, G. Yovel, N. Rosenzweig, A. London, J. Ruckh, K. W. Kim, E. Klein, V. Kalchenko, P. Bendel, S. A. Lira, S. Jung, and M. Schwartz. 2013. Recruitment of beneficial M2 macrophages to injured spinal cord is orchestrated by remote brain choroid plexus. Immunity 38: 555-569. 
13. Segura, E., and S. Amigorena. 2013. Inflammatory dendritic cells in mice and humans. Trends Immunol 34: 440-445.

14. Xiong, H., and E. G. Pamer. 2015. Monocytes and infection: modulator, messenger and effector. Immunobiology 220: 210-214.

15. Duan, M., M. L. Hibbs, and W. Chen. 2016. The contributions of lung macrophage and monocyte heterogeneity to influenza pathogenesis. Immunol Cell Biol.

16. Guilliams, M., F. Ginhoux, C. Jakubzick, S. H. Naik, N. Onai, B. U. Schraml, E. Segura, R. Tussiwand, and S. Yona. 2014. Dendritic cells, monocytes and macrophages: a unified nomenclature based on ontogeny. Nat Rev Immunol 14: 571-578.

17. Channappanavar, R., A. R. Fehr, R. Vijay, M. Mack, J. Zhao, D. K. Meyerholz, and S. Perlman. 2016. Dysregulated Type I Interferon and Inflammatory MonocyteMacrophage Responses Cause Lethal Pneumonia in SARS-CoV-Infected Mice. Cell Host Microbe 19: 181-193.

18. Gralinski, L. E., and R. S. Baric. 2015. Molecular pathology of emerging coronavirus infections. J Pathol 235: 185-195.

19. Israelow, B., E. Song, T. Mao, P. Lu, A. Meir, F. Liu, M. M. Alfajaro, J. Wei, H. Dong, R. J. Homer, A. Ring, C. B. Wilen, and A. Iwasaki. 2020. Mouse model of SARS-CoV-2 reveals inflammatory role of type I interferon signaling. J Exp Med 217.

20. Dawson, T. C., M. A. Beck, W. A. Kuziel, F. Henderson, and N. Maeda. 2000. Contrasting effects of CCR5 and CCR2 deficiency in the pulmonary inflammatory response to influenza A virus. Am J Pathol 156: 1951-1959.

21. Aldridge, J. R., Jr., C. E. Moseley, D. A. Boltz, N. J. Negovetich, C. Reynolds, J. Franks, S. A. Brown, P. C. Doherty, R. G. Webster, and P. G. Thomas. 2009. TNF/iNOS-producing dendritic cells are the necessary evil of lethal influenza virus infection. Proc Natl Acad Sci U S A 106: 5306-5311.

22. Lin, S. J., M. Lo, R. L. Kuo, S. R. Shih, D. M. Ojcius, J. Lu, C. K. Lee, H. C. Chen, M. Y. Lin, C. M. Leu, C. N. Lin, and C. H. Tsai. 2014. The pathological effects of CCR2+ inflammatory monocytes are amplified by an IFNAR1-triggered chemokine feedback loop in highly pathogenic influenza infection. J Biomed Sci 21: 99.

23. Herold, S., M. Steinmueller, W. von Wulffen, L. Cakarova, R. Pinto, S. Pleschka, M. Mack, W. A. Kuziel, N. Corazza, T. Brunner, W. Seeger, and J. Lohmeyer. 2008. Lung epithelial apoptosis in influenza virus pneumonia: the role of macrophage-expressed TNFrelated apoptosis-inducing ligand. J Exp Med 205: 3065-3077.

24. Muller, U., U. Steinhoff, L. F. Reis, S. Hemmi, J. Pavlovic, R. M. Zinkernagel, and M. Aguet. 1994. Functional role of type I and type II interferons in antiviral defense. Science 264: 1918-1921.

25. McNab, F., K. Mayer-Barber, A. Sher, A. Wack, and A. O'Garra. 2015. Type I interferons in infectious disease. Nat Rev Immunol 15: 87-103.

26. Davidson, S., S. Crotta, T. M. McCabe, and A. Wack. 2014. Pathogenic potential of interferon alphabeta in acute influenza infection. Nat Commun 5: 3864.

27. Frieman, M. B., J. Chen, T. E. Morrison, A. Whitmore, W. Funkhouser, J. M. Ward, E. W. Lamirande, A. Roberts, M. Heise, K. Subbarao, and R. S. Baric. 2010. SARS-CoV pathogenesis is regulated by a STAT1 dependent but a type I, II and III interferon receptor independent mechanism. PLoS Pathog 6: e1000849.

28. Mahlakoiv, T., D. Ritz, M. Mordstein, M. L. DeDiego, L. Enjuanes, M. A. Muller, C. Drosten, and P. Staeheli. 2012. Combined action of type I and type III interferon 
restricts initial replication of severe acute respiratory syndrome coronavirus in the lung but fails to inhibit systemic virus spread. J Gen Virol 93: 2601-2605.

29. Staeheli, P., R. Grob, E. Meier, J. G. Sutcliffe, and O. Haller. 1988. Influenza virussusceptible mice carry Mx genes with a large deletion or a nonsense mutation. Mol Cell Biol 8: 4518-4523.

30. Mordstein, M., G. Kochs, L. Dumoutier, J. C. Renauld, S. R. Paludan, K. Klucher, and P. Staeheli. 2008. Interferon-lambda contributes to innate immunity of mice against influenza A virus but not against hepatotropic viruses. PLoS Pathog 4: e1000151.

31. Steinhauer, D. A. 1999. Role of hemagglutinin cleavage for the pathogenicity of influenza virus. Virology 258: 1-20.

32. Garcia-Sastre, A., R. K. Durbin, H. Zheng, P. Palese, R. Gertner, D. E. Levy, and J. E. Durbin. 1998. The role of interferon in influenza virus tissue tropism. J Virol 72: 85508558.

33. Szretter, K. J., S. Gangappa, J. A. Belser, H. Zeng, H. Chen, Y. Matsuoka, S. Sambhara, D. E. Swayne, T. M. Tumpey, and J. M. Katz. 2009. Early control of H5N1 influenza virus replication by the type I interferon response in mice.J Virol 83: 5825-5834.

34. Salomon, R., E. Hoffmann, and R. G. Webster. 2007. Inhibition of the cytokine response does not protect against lethal H5N1 influenza infection. Proc Natl Acad Sci U S A 104: 12479-12481.

35. Uccellini, M. B., and A. Garcia-Sastre. 2018. ISRE-Reporter Mouse Reveals High Basal and Induced Type I IFN Responses in Inflammatory Monocytes. Cell Rep 25: 27842796 e2783.

36. Seo, S. U., H. J. Kwon, H. J. Ko, Y. H. Byun, B. L. Seong, S. Uematsu, S. Akira, and M. N. Kweon. 2011. Type I interferon signaling regulates Ly6C(hi) monocytes and neutrophils during acute viral pneumonia in mice. PLoS Pathog 7: e1001304.

37. Cole, S. L., J. Dunning, W. L. Kok, K. H. Benam, A. Benlahrech, E. Repapi, F. O. Martinez, L. Drumright, T. J. Powell, M. Bennett, R. Elderfield, C. Thomas, M. investigators, T. Dong, J. McCauley, F. Y. Liew, S. Taylor, M. Zambon, W. Barclay, V. Cerundolo, P. J. Openshaw, A. J. McMichael, and L. P. Ho. 2017. M1-like monocytes are a major immunological determinant of severity in previously healthy adults with lifethreatening influenza. JCI Insight 2: e91868.

38. Ellis, G. T., S. Davidson, S. Crotta, N. Branzk, V. Papayannopoulos, and A. Wack. 2015. TRAIL+ monocytes and monocyte-related cells cause lung damage and thereby increase susceptibility to influenza-Streptococcus pneumoniae coinfection. EMBO Rep 16: 1203-1218.

39. Steel, J., A. C. Lowen, L. Pena, M. Angel, A. Solorzano, R. Albrecht, D. R. Perez, A. GarciaSastre, and P. Palese. 2009. Live attenuated influenza viruses containing NS1 truncations as vaccine candidates against H5N1 highly pathogenic avian influenza. $J$ Virol 83: 1742-1753.

40. Nakaya, H. I., J. Wrammert, E. K. Lee, L. Racioppi, S. Marie-Kunze, W. N. Haining, A. R. Means, S. P. Kasturi, N. Khan, G. M. Li, M. McCausland, V. Kanchan, K. E. Kokko, S. Li, R. Elbein, A. K. Mehta, A. Aderem, K. Subbarao, R. Ahmed, and B. Pulendran. 2011. Systems biology of vaccination for seasonal influenza in humans. Nat Immunol 12: 786-795.

41. Rieckmann, J. C., R. Geiger, D. Hornburg, T. Wolf, K. Kveler, D. Jarrossay, F. Sallusto, S. S. Shen-Orr, A. Lanzavecchia, M. Mann, and F. Meissner. 2017. Social network 
architecture of human immune cells unveiled by quantitative proteomics. Nat Immunol 18: 583-593.

42. Peiris, J. S., C. Y. Cheung, C. Y. Leung, and J. M. Nicholls. 2009. Innate immune responses to influenza A H5N1: friend or foe? Trends Immunol 30: 574-584.

43. Gambotto, A., S. M. Barratt-Boyes, M. D. de Jong, G. Neumann, and Y. Kawaoka. 2008. Human infection with highly pathogenic H5N1 influenza virus. Lancet 371: 14641475.

44. Pang, I. K., P. S. Pillai, and A. Iwasaki. 2013. Efficient influenza A virus replication in the respiratory tract requires signals from TLR7 and RIG-I. Proc Natl Acad Sci U S A 110: 13910-13915.

45. Fujikura, D., S. Chiba, D. Muramatsu, M. Kazumata, Y. Nakayama, T. Kawai, S. Akira, H. Kida, and T. Miyazaki. 2013. Type-I interferon is critical for FasL expression on lung cells to determine the severity of influenza. PLoS One 8: e55321.

46. Legge, K. L., and T. J. Braciale. 2005. Lymph node dendritic cells control CD8+ T cell responses through regulated FasL expression. Immunity 23: 649-659.

47. Bardina, S. V., D. Michlmayr, K. W. Hoffman, C. J. Obara, J. Sum, I. F. Charo, W. Lu, A. G. Pletnev, and J. K. Lim. 2015. Differential Roles of Chemokines CCL2 and CCL7 in Monocytosis and Leukocyte Migration during West Nile Virus Infection. J Immunol 195: 4306-4318.

48. Goritzka, M., S. Makris, F. Kausar, L. R. Durant, C. Pereira, Y. Kumagai, F. J. Culley, M. Mack, S. Akira, and C. Johansson. 2015. Alveolar macrophage-derived type I interferons orchestrate innate immunity to RSV through recruitment of antiviral monocytes. J Exp Med 212: 699-714.

49. Perrone, L. A., J. K. Plowden, A. Garcia-Sastre, J. M. Katz, and T. M. Tumpey. 2008. H5N1 and 1918 pandemic influenza virus infection results in early and excessive infiltration of macrophages and neutrophils in the lungs of mice. PLoS Pathog 4: e1000115.

50. Leung, Y. H., J. M. Nicholls, C. K. Ho, S. F. Sia, C. K. Mok, S. A. Valkenburg, P. Cheung, K. P. Hui, R. W. Chan, Y. Guan, S. Akira, and J. S. Peiris. 2014. Highly pathogenic avian influenza A H5N1 and pandemic H1N1 virus infections have different phenotypes in Toll-like receptor 3 knockout mice. J Gen Virol 95: 1870-1879.

51. van den Brand, J. M., B. L. Haagmans, D. van Riel, A. D. Osterhaus, and T. Kuiken. 2014. The pathology and pathogenesis of experimental severe acute respiratory syndrome and influenza in animal models. J Comp Pathol 151: 83-112.

52. Szretter, K. J., S. Gangappa, X. Lu, C. Smith, W. J. Shieh, S. R. Zaki, S. Sambhara, T. M. Tumpey, and J. M. Katz. 2007. Role of host cytokine responses in the pathogenesis of avian H5N1 influenza viruses in mice. J Virol 81: 2736-2744.

53. Coates, B. M., K. L. Staricha, C. M. Koch, Y. Cheng, D. K. Shumaker, G. R. S. Budinger, H. Perlman, A. V. Misharin, and K. M. Ridge. 2018. Inflammatory Monocytes Drive Influenza A Virus-Mediated Lung Injury in Juvenile Mice. J Immunol 200: 2391-2404.

54. Ghoneim, H. E., P. G. Thomas, and J. A. McCullers. 2013. Depletion of alveolar macrophages during influenza infection facilitates bacterial superinfections. $J$ Immunol 191: 1250-1259.

55. Nakajima, N., N. Van Tin, Y. Sato, H. N. Thach, H. Katano, P. H. Diep, T. Kumasaka, N. T. Thuy, H. Hasegawa, L. T. San, S. Kawachi, N. T. Liem, K. Suzuki, and T. Sata. 2013. Pathological study of archival lung tissues from five fatal cases of avian H5N1 influenza in Vietnam. Mod Pathol 26: 357-369. 
56. Liao, M., Y. Liu, J. Yuan, Y. Wen, G. Xu, J. Zhao, L. Cheng, J. Li, X. Wang, F. Wang, L. Liu, I. Amit, S. Zhang, and Z. Zhang. 2020. Single-cell landscape of bronchoalveolar immune cells in patients with COVID-19. Nat Med 26: 842-844.

57. Lee, A. C. Y., K. K. W. To, H. Zhu, H. Chu, C. Li, W. W. N. Mak, A. J. X. Zhang, and K. Y. Yuen. 2017. Avian influenza virus A H7N9 infects multiple mononuclear cell types in peripheral blood and induces dysregulated cytokine responses and apoptosis in infected monocytes. J Gen Virol 98: 922-934.

58. Cline, T. D., E. A. Karlsson, B. J. Seufzer, and S. Schultz-Cherry. 2013. The hemagglutinin protein of highly pathogenic H5N1 influenza viruses overcomes an early block in the replication cycle to promote productive replication in macrophages. J Virol 87: 14111419.

59. Barbalat, R., L. Lau, R. M. Locksley, and G. M. Barton. 2009. Toll-like receptor 2 on inflammatory monocytes induces type I interferon in response to viral but not bacterial ligands. Nat Immunol 10: 1200-1207.

60. Lee, P. Y., J. S. Weinstein, D. C. Nacionales, P. O. Scumpia, Y. Li, E. Butfiloski, N. van Rooijen, L. Moldawer, M. Satoh, and W. H. Reeves. 2008. A novel type I IFN-producing cell subset in murine lupus. J Immunol 180: 5101-5108.

61. Brincks, E. L., A. Katewa, T. A. Kucaba, T. S. Griffith, and K. L. Legge. 2008. CD8 T cells utilize TRAIL to control influenza virus infection. J Immunol 181: 4918-4925.

62. Wurzer, W. J., C. Ehrhardt, S. Pleschka, F. Berberich-Siebelt, T. Wolff, H. Walczak, O. Planz, and S. Ludwig. 2004. NF-kappaB-dependent induction of tumor necrosis factor-related apoptosis-inducing ligand (TRAIL) and Fas/FasL is crucial for efficient influenza virus propagation. J Biol Chem 279: 30931-30937.

63. Terry, R. L., D. R. Getts, C. Deffrasnes, C. van Vreden, I. L. Campbell, and N. J. King. 2012. Inflammatory monocytes and the pathogenesis of viral encephalitis. $J$ Neuroinflammation 9: 270.

64. Stifter, S. A., N. Bhattacharyya, R. Pillay, M. Florido, J. A. Triccas, W. J. Britton, and C. G. Feng. 2016. Functional Interplay between Type I and II Interferons Is Essential to Limit Influenza A Virus-Induced Tissue Inflammation. PLoS Pathog 12: e1005378.

65. Thomas, G., R. Tacke, C. C. Hedrick, and R. N. Hanna. 2015. Nonclassical patrolling monocyte function in the vasculature. Arterioscler Thromb Vasc Biol 35: 1306-1316.

66. Hanna, R. N., L. M. Carlin, H. G. Hubbeling, D. Nackiewicz, A. M. Green, J. A. Punt, F. Geissmann, and C. C. Hedrick. 2011. The transcription factor NR4A1 (Nur77) controls bone marrow differentiation and the survival of Ly6C- monocytes. Nat Immunol 12: 778-785.

67. Lee, P. Y., Y. Li, Y. Kumagai, Y. Xu, J. S. Weinstein, E. S. Kellner, D. C. Nacionales, E. J. Butfiloski, N. van Rooijen, S. Akira, E. S. Sobel, M. Satoh, and W. H. Reeves. 2009. Type I interferon modulates monocyte recruitment and maturation in chronic inflammation. Am J Pathol 175: 2023-2033.

68. Cros, J., N. Cagnard, K. Woollard, N. Patey, S. Y. Zhang, B. Senechal, A. Puel, S. K. Biswas, D. Moshous, C. Picard, J. P. Jais, D. D'Cruz, J. L. Casanova, C. Trouillet, and F. Geissmann. 2010. Human CD14dim monocytes patrol and sense nucleic acids and viruses via TLR7 and TLR8 receptors. Immunity 33: 375-386.

69. Carlin, L. M., E. G. Stamatiades, C. Auffray, R. N. Hanna, L. Glover, G. Vizcay-Barrena, C. C. Hedrick, H. T. Cook, S. Diebold, and F. Geissmann. 2013. Nr4a1-dependent 
Ly6C(low) monocytes monitor endothelial cells and orchestrate their disposal. Cell 153: 362-375.

70. Peng, Y., Y. Latchman, and K. B. Elkon. 2009. Ly6C(low) monocytes differentiate into dendritic cells and cross-tolerize T cells through PDL-1. J Immunol 182: 2777-2785.

71. Hou, W., J. S. Gibbs, X. Lu, C. B. Brooke, D. Roy, R. L. Modlin, J. R. Bennink, and J. W. Yewdell. 2012. Viral infection triggers rapid differentiation of human blood monocytes into dendritic cells. Blood 119: 3128-3131.

72. Cao, W., A. K. Taylor, R. E. Biber, W. G. Davis, J. H. Kim, A. J. Reber, T. Chirkova, J. A. De La Cruz, A. Pandey, P. Ranjan, J. M. Katz, S. Gangappa, and S. Sambhara. 2012. Rapid differentiation of monocytes into type I IFN-producing myeloid dendritic cells as an antiviral strategy against influenza virus infection. J Immunol 189: 2257-2265.

73. Santini, S. M., C. Lapenta, M. Logozzi, S. Parlato, M. Spada, T. Di Pucchio, and F. Belardelli. 2000. Type I interferon as a powerful adjuvant for monocyte-derived dendritic cell development and activity in vitro and in Hu-PBL-SCID mice. J Exp Med 191: 1777-1788.

74. Goudot, C., A. Coillard, A. C. Villani, P. Gueguen, A. Cros, S. Sarkizova, T. L. Tang-Huau, M. Bohec, S. Baulande, N. Hacohen, S. Amigorena, and E. Segura. 2017. Aryl Hydrocarbon Receptor Controls Monocyte Differentiation into Dendritic Cells versus Macrophages. Immunity 47: 582-596 e586.

75. Soudja, S. M., A. L. Ruiz, J. C. Marie, and G. Lauvau. 2012. Inflammatory monocytes activate memory CD8(+) T and innate NK lymphocytes independent of cognate antigen during microbial pathogen invasion. Immunity 37: 549-562.

76. Lee, A. J., B. Chen, M. V. Chew, N. G. Barra, M. M. Shenouda, T. Nham, N. van Rooijen, M. Jordana, K. L. Mossman, R. D. Schreiber, M. Mack, and A. A. Ashkar. 2017. Inflammatory monocytes require type I interferon receptor signaling to activate NK cells via IL-18 during a mucosal viral infection. J Exp Med 214: 1153-1167.

77. Dominguez-Andres, J., L. Feo-Lucas, M. Minguito de la Escalera, L. Gonzalez, M. LopezBravo, and C. Ardavin. 2017. Inflammatory Ly6Chigh Monocytes Protect against Candidiasis through IL-15-Driven NK Cell/Neutrophil Activation. Immunity 46: 10591072 e1054.

78. Kumagai, Y., O. Takeuchi, H. Kato, H. Kumar, K. Matsui, E. Morii, K. Aozasa, T. Kawai, and S. Akira. 2007. Alveolar macrophages are the primary interferon-alpha producer in pulmonary infection with RNA viruses. Immunity 27: 240-252.

79. Fourati, S., A. Talla, M. Mahmoudian, J. G. Burkhart, R. Klen, R. Henao, T. Yu, Z. Aydin, K. Y. Yeung, M. E. Ahsen, R. Almugbel, S. Jahandideh, X. Liang, T. E. M. Nordling, M. Shiga, A. Stanescu, R. Vogel, D. C. C. Respiratory Viral, G. Pandey, C. Chiu, M. T. McClain, C. W. Woods, G. S. Ginsburg, L. L. Elo, E. L. Tsalik, L. M. Mangravite, and S. K. Sieberts. 2018. A crowdsourced analysis to identify ab initio molecular signatures predictive of susceptibility to viral infection. Nat Commun 9: 4418.

80. Rusinova, I., S. Forster, S. Yu, A. Kannan, M. Masse, H. Cumming, R. Chapman, and P. J. Hertzog. 2013. Interferome v2.0: an updated database of annotated interferonregulated genes. Nucleic Acids Res 41: D1040-1046.

81. Newman, A. M., C. L. Liu, M. R. Green, A. J. Gentles, W. Feng, Y. Xu, C. D. Hoang, M. Diehn, and A. A. Alizadeh. 2015. Robust enumeration of cell subsets from tissue expression profiles. Nat Methods 12: 453-457. 
82. Misharin, A. V., L. Morales-Nebreda, P. A. Reyfman, C. M. Cuda, J. M. Walter, A. C. McQuattie-Pimentel, C. I. Chen, K. R. Anekalla, N. Joshi, K. J. N. Williams, H. AbdalaValencia, T. J. Yacoub, M. Chi, S. Chiu, F. J. Gonzalez-Gonzalez, K. Gates, A. P. Lam, T. T. Nicholson, P. J. Homan, S. Soberanes, S. Dominguez, V. K. Morgan, R. Saber, A. Shaffer, M. Hinchcliff, S. A. Marshall, A. Bharat, S. Berdnikovs, S. M. Bhorade, E. T. Bartom, R. I. Morimoto, W. E. Balch, J. I. Sznajder, N. S. Chandel, G. M. Mutlu, M. Jain, C. J. Gottardi, B. D. Singer, K. M. Ridge, N. Bagheri, A. Shilatifard, G. R. S. Budinger, and H. Perlman. 2017. Monocyte-derived alveolar macrophages drive lung fibrosis and persist in the lung over the life span. J Exp Med 214: 2387-2404.

83. Mould, K. J., L. Barthel, M. P. Mohning, S. M. Thomas, A. L. McCubbrey, T. Danhorn, S. M. Leach, T. E. Fingerlin, B. P. O'Connor, J. A. Reisz, A. D'Alessandro, D. L. Bratton, C. V. Jakubzick, and W. J. Janssen. 2017. Cell Origin Dictates Programming of Resident versus Recruited Macrophages during Acute Lung Injury. Am J Respir Cell Mol Biol 57: 294-306.

84. Park, C., S. Li, E. Cha, and C. Schindler. 2000. Immune response in Stat2 knockout mice. Immunity 13: 795-804.

85. Bourmakina, S. V., and A. Garcia-Sastre. 2005. The morphology and composition of influenza A virus particles are not affected by low levels of M1 and M2 proteins in infected cells. J Virol 79: 7926-7932. 